\title{
A GENERALIZED CLASS OF EXPONENTIATED MODI ED WEIBULL DISTRIBUTION WITH APPLICATIONS
}

\author{
Shusen $\mathrm{Pu}^{1}$; Broderick O. Oluyede ${ }^{2}$, Yuqi Qiu ${ }^{3}$ and Daniel Linder ${ }^{4}$
}

\begin{abstract}
In this paper, a new class of five parameter gamma-exponentiated or generalized modified Weibull (GEMW) distribution which includes exponential, Rayleigh, Weibull, modified Weibull, exponentiated Weibull, exponentiated exponential, exponentiated modified Weibull, exponentiated modified exponential, gamma-exponentiated exponential, gammaexponentiated Rayleigh, gamma-modified Weibull, gamma-modified exponential, gamma-Weibull, gamma-Rayleigh and gamma-exponential distributions as special cases is proposed and studied. Mathematical properties of this new class of distributions including moments, mean deviations, Bonferroni and Lorenz curves, distribution of order statistics and Renyi entropy are presented. Maximum likelihood estimation technique is used to estimate the model parameters and applications to real data sets presented in order to illustrate the usefulness of this new class of distributions and its sub-models.
\end{abstract}

Key words: Modified Weibull distribution; statistical properties; maximum likelihood; applications.

\section{Introduction}

Weibull distribution (Weibull, 1951) has exponential and Rayleigh as special sub-models and it is one of the most popular distributions for modeling lifetime data with monotone failure rates. However, as for non-monotone failure rates, Weibull distribution does not $t$ very well. Recently, several modified Weibull distributions with additional parameters have been proposed and studied as lifetime distributions in reliability and lifetime data analysis.

Recently, several ways of generating new probability distributions from classic ones have been developed and discussed in the literature on distribution theory and its applications. Nelson (1982) stated that distributions with bathtub-shaped failure rate are complex and, therefore, di cult to model. The distribution pro-posed by Hjorth (1980) is an example. Rajarshi and Rajarshi (1988) presented a revision of these distributions, and Haupt and Schabe (1992) introduced a new lifetime model with bathtub-shaped failure rates. However, these models are not sufficient to address the various complex practical situations, so new classes of distributions were presented based on the modifications of the Weibull distribution to satisfy non-monotonic failure rate. For a review of these models, the reader can refer to Pham and Lai (2007), where the authors summarized some generalizations of Weibull distribution. Jones (2004) studied a family of distributions derived from the distribution of order statistics, the beta-generated family proposed by Eugene et al. (2002). Other generalizations include: the exponentiated Weibull (EW) (Gupta and Kundu, 1999), the modified Weibull (MW) (Lai et al., 2003), the beta exponential (BE) (Nadarajah and Kotz, 
2006). Some re-cent extensions are the generalized modified Weibull (GMW) (Carrasco et al., 2008), the beta modi ed Weibull (BMW) (Silva et al., 2010), the Weibull-G fam-ily (Burguignon et al., 2014), the Gamma-exponentiated Weibull distributions (GEW) (Pinho et al., 2012) and the McDonald exponentiated modified Weibull (McEMW) (Merovci and Elbatal, 2015).

Ristic and Balakrishnan (2011), provided a new family of distributions whose cumulative distribution function (cdf) were generated by equation (1). As a natural extension, in this paper we introduce a new distribution with ve pa-rameters, referred to as the gammaexponentiated modi ed Weibull (GEMW) distribution with the aim of attracting wider application in reliability, biology and other areas of research. This generalization contains as special sub-models several distributions such as the EW (Gupta and Kundu, 1999), MW, generalized Rayleigh (GR) (Kundu and Rekab, 2005) and a new sub-model, namely Gamma modi ed Weibull (GMW) distributions, along with several others. Due to its exibility in accommodating all the forms of the hazard function, the proposed GEMW distribution seems to be an important distribution that can be used in various problems in modeling survival data. The GEMW distribution is not only useful for modeling bathtub-shaped failure rate data but also suitable for testing goodness-of- $t$ of some special sub-models such as the EW (Gupta and Kundu, 1999), MW, GMW (new) and GEW distributions.

The rest of the paper is organized as follows. In Section 2; we de ne the GEMW distribution and provide its hazard rate, reverse hazard and quantile functions. Expansions for its probability density function (pdf) and some special sub-models are presented as well. The moments, moment generating and characteristic functions are given in section 3: Section 4 is devoted to mean deviations about the mean and the median, Bonferroni and Lorenz curves. Section 5 contains results on the distribution of order statistics and Renyi entropy. In section 6; estimation of the parameters of the GEMW distribution via the method of maximum likelihood is presented. Applications are given in section 7, followed by concluding remarks in section 8:

\section{The Model}

\subsection{Definition}

Based on a continuous cdf $F(x)$ with survival function $\bar{F}(x)$ and $\operatorname{pdf} f(x)$ and the method proposed by Zografos and Balakrishnan (2009), Ristic and Balakrishnan (2011) proposed an alternative gamma-generator defined by the cdf and pdf:

$$
G(x)=1-\frac{1}{\Gamma(\delta)} \int_{0}^{-\log F(x)} t^{\delta-1} e^{-t} d t, \quad x \in \mathbf{R}, \delta>0,
$$

and

$$
g(x)=\frac{1}{\Gamma(\delta)}[-\log F(x)]^{\delta-1} f(x),
$$

respectively. For $\delta=n \in \mathrm{N}$, equation (2) is the pdf of the $n^{\text {th }}$ lower record value of a sequence of i.i.d. variables from a population with density $f(x)$. 
Consider the exponentiated modified Weibull (EMW) (Carrasco et al., 2008) distribution with cdf given by

$$
F(x)=\left[1-e^{-\left(\frac{x}{\lambda}\right)^{k} e^{\beta x}}\right]^{\alpha},
$$

and pdf

$$
f(x)=(k+\beta x) \frac{\alpha}{\lambda}\left(\frac{x}{\lambda}\right)^{k-1} e^{-\left(\frac{x}{\lambda}\right)^{k} e^{\beta x}+\beta x}\left[1-e^{-\left(\frac{x}{\lambda}\right)^{k} e^{\beta x}}\right]^{\alpha-1},
$$

where $x, k, \alpha, \lambda>0$ and $\beta \geq 0$. By replacing $F(x)$ in (1) by the EMW cdf, we obtain a new extension of EMW distribution, called the gamma-exponentiated modified Weibull (GEMW) distribution. Inserting (3) in (1) yields the GEMW cdf (for $x>0$ )

$$
G(x)=1-\frac{\gamma\left\{\delta,-\log \left[1-e^{-\left(\frac{\pi}{\lambda}\right)^{k} e^{\beta x}}\right]^{\alpha}\right\}}{\Gamma(\delta)},
$$
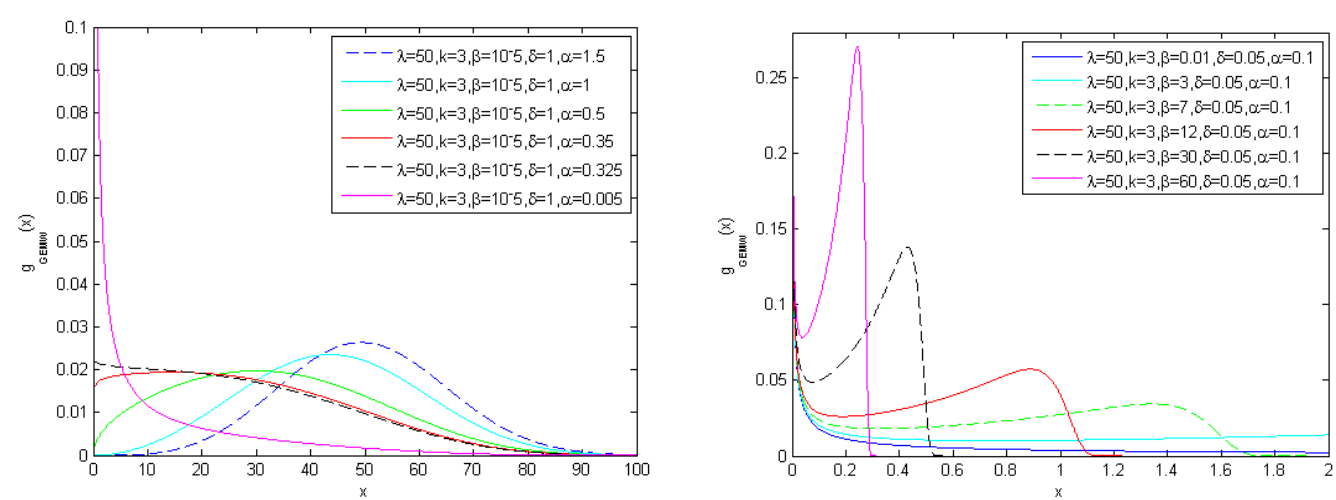

Figure 1: Graphs of GEMW pdf with varying values of $\alpha$ and $\beta$; respectively
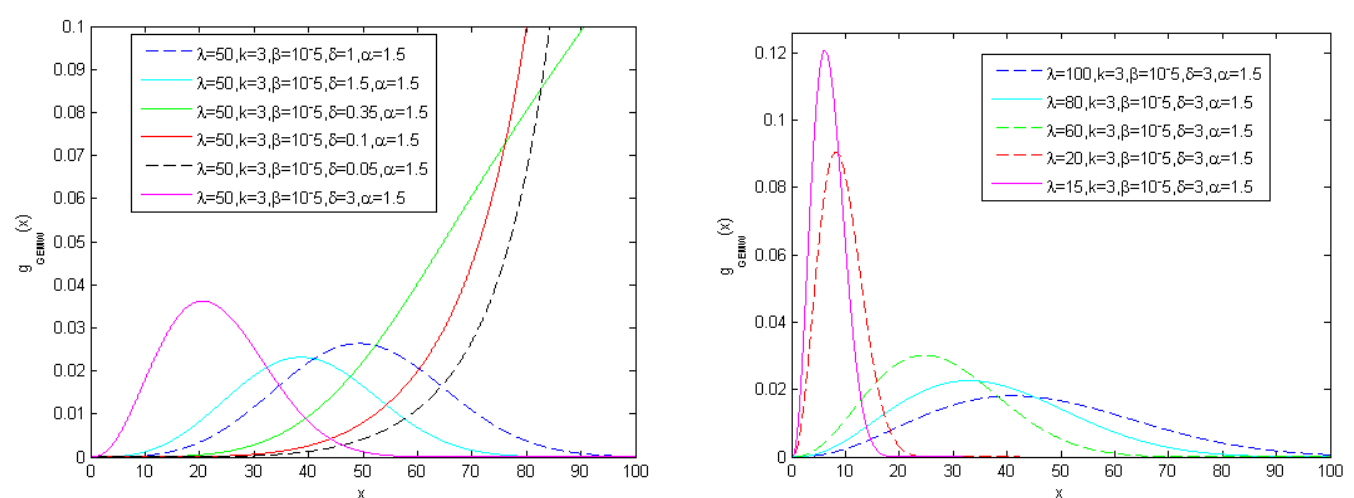

Figure 2: Graphs of GEMW pdf with varying values of $\delta$ and $\lambda$; respectively

where $, k, \alpha, \lambda>0, \beta \geq 0$ and $\gamma(\delta, x)=\int_{0}^{x} t^{\delta-1} e^{-t} d t$ is the incomplete gamma function. The pdf corresponding to (5) is given by 


$$
\begin{aligned}
g(x) & =\frac{1}{\Gamma(\delta)}\left(-\log \left[1-e^{-\left(\frac{x}{\lambda}\right)^{k} e^{\beta x}}\right]^{\alpha}\right)^{\delta-1}(k+\beta x) \\
& \times\left(\frac{\alpha}{\lambda}\right)\left(\frac{x}{\lambda}\right)^{k-1} e^{-\left(\frac{x}{\lambda}\right)^{k} e^{\beta x}+\beta x}\left[1-e^{-\left(\frac{x}{\lambda}\right)^{k} e^{\beta x}}\right]^{\alpha-1},
\end{aligned}
$$

for $x, k, \alpha, \lambda>0$ and $\beta \geq 0$. A random variable $\mathrm{X}$ having density (6) is denoted by $X \sim G E M$ $W(\delta, \alpha, \beta, k, \lambda)$. Several possible shapes of the GEMW pdf are shown in Figure 1, 2 and 3. Note that the parameters $\lambda$ controls the scale of the distribution, $k, \alpha$ and $\delta$ controls its shape, $\beta$ is a sort of accelerating factor in the imperfection time that works as a factor of fragility in the survival of the individual as time increases.

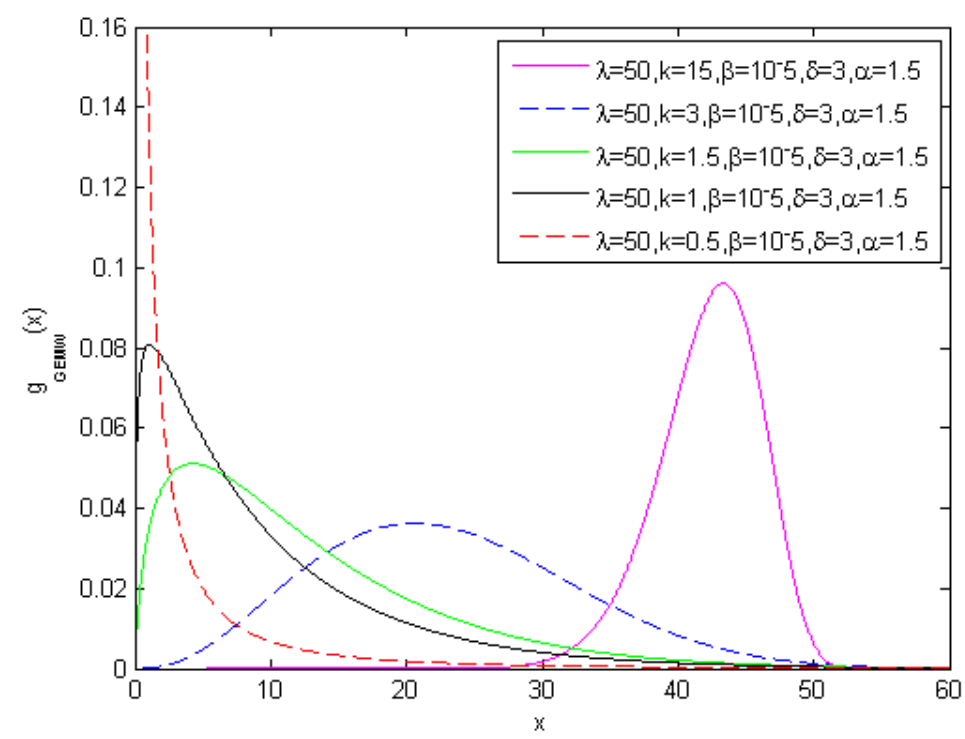

Figure 3: Graph of GEMW pdf when only the parameter $k$ changes

\subsection{Hazard rate, reverse hazard and quantile functions}

In this section, we provide the hazard rate, reverse hazard and quantile functions of the GEMW distribution.

\subsubsection{Hazard rate and reverse hazard functions}

Note that if $X$ is a continuous random variable with $\operatorname{cdf} G(x)$; and $\operatorname{pdf} g(x)$; then the failure of hazard rate function (hrf), reverse hazard function (rhf) and mean residual life functions are given by $h_{G}(x)=\frac{g(x)}{\bar{G}(x)}, \tau_{G}(x)=\frac{g(x)}{\mathrm{G}(\mathrm{x})}$; and $\delta_{G}(x)=\int_{x}^{\infty} \bar{G}(u) d u / \bar{G}(u)$, respectively. The functions $h_{G}(x), \delta_{G}(x)$, and $\bar{G}(x)$ are equivalent. (Shaked and Shanthikumar, 1994). The hazard rate and reverse hazard rate functions of GEMW distribution are given by 


$$
h_{G}(x)=\frac{\left[-\log \left[1-e^{-\left(\frac{x}{\lambda}\right)^{k} e^{\beta x}}\right]^{\alpha}\right]^{\delta-1}(k+\beta x) \frac{\alpha}{\lambda}\left(\frac{x}{\lambda}\right)^{k-1} e^{-\left(\frac{x}{\lambda}\right)^{k} e^{\beta x}+\beta x}\left[1-e^{-\left(\frac{x}{\lambda}\right)^{k} e^{\beta x}}\right]^{\alpha-1}}{\gamma\left\{-\log \left[1-e^{-\left(\frac{x}{\lambda}\right)^{k} e^{\beta x}}\right]^{\alpha}, \delta\right\}},
$$

and

$$
\tau_{G}(x)=\frac{\left[-\log \left[1-e^{-\left(\frac{x}{\lambda}\right)^{k} e^{\beta x}}\right]^{\alpha}\right]^{\delta-1}(k+\beta x) \frac{\alpha}{\lambda}\left(\frac{x}{\lambda}\right)^{k-1} e^{-\left(\frac{x}{\lambda}\right)^{k} e^{\beta x}+\beta x}\left[1-e^{-\left(\frac{x}{\lambda}\right)^{k} e^{\beta x}}\right]^{\alpha-1}}{\Gamma(\delta)-\gamma\left\{-\log \left[1-e^{-\left(\frac{x}{\lambda}\right)^{k} e^{\beta x}}\right]^{\alpha}, \delta\right\}},
$$

respectively
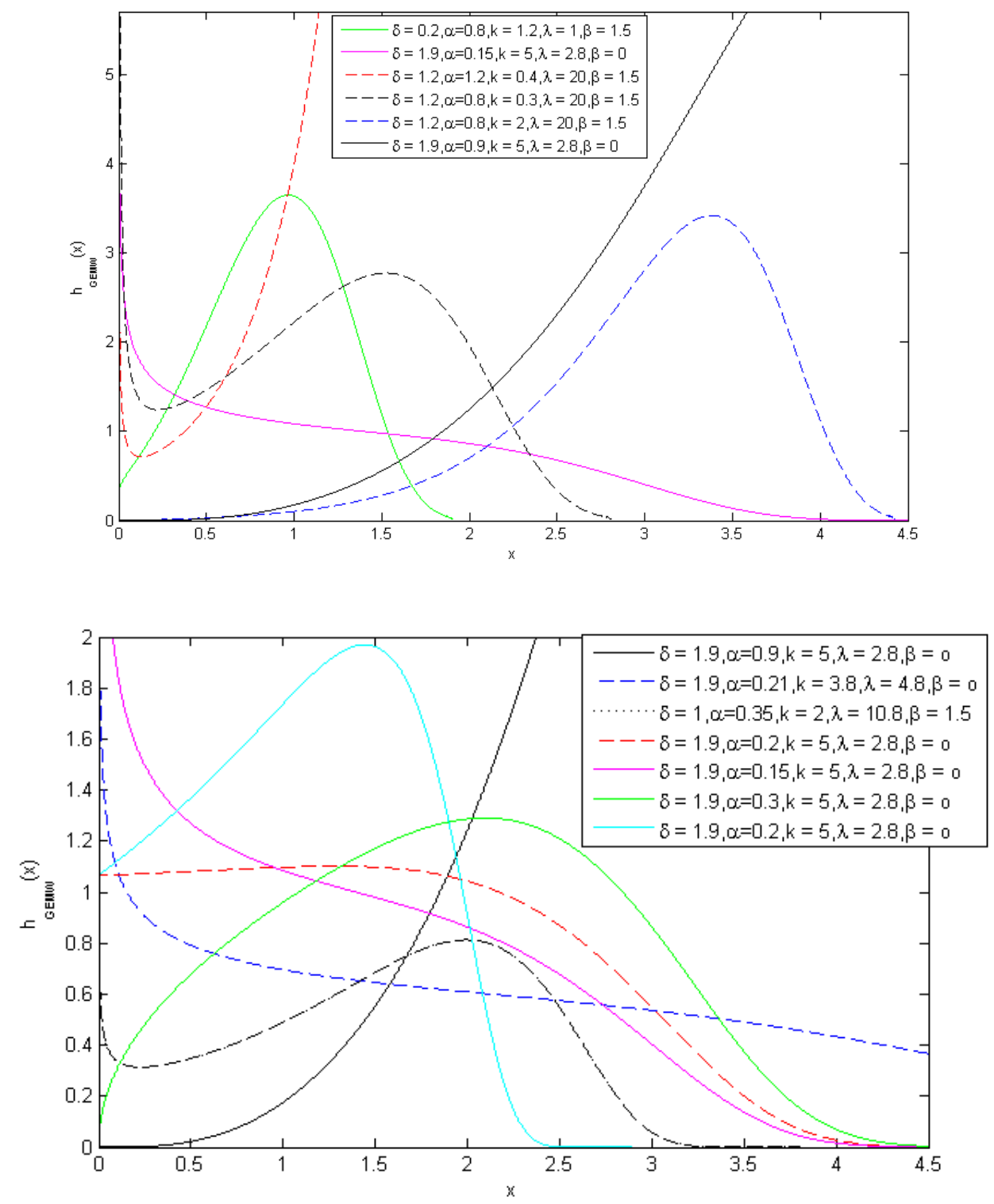

Figure 4: Graphs of GEMW hazard function

Plots of hrf are presented in Figure 4. These plots show various shapes including monotonically decreasing, monotonically increasing, unimodal and bathtub shapes for different combinations of the values of the parameters. This flexibility makes the GEMW hrf 
suitable for monotonic and non-monotonic empirical hazard behaviors which are more likely to be the case in real life situations.

The density and hazard functions can exhibit different behavior depending on the values of the parameters when chosen to be positive, as shown in these plots. However, it is hard to analyze the shape of both the density and hazard function due to their complicated forms.

\subsubsection{Quantile function}

We can know more about this model by expanding the density function and analyzing the quantile function. The GEMW quantile function can be obtained by inverting $\bar{G}(x)=1-u$, where $G(x)=u$ and

$$
\bar{G}(x)=\frac{\gamma\left\{-\log \left[1-e^{-\left(\frac{x}{\lambda}\right)^{k} e^{\beta x}}\right]^{\alpha}, \delta\right\}}{\Gamma(\delta)} .
$$

Let $z=\gamma^{-1}[(1-u) \Gamma(\delta), \delta]$, then one can get,

$$
\log \left(\log \left(1-e^{-\frac{z}{\alpha}}\right)\right)+k \log (x)+k \log (\lambda)+\beta x=0,
$$

and the inverse incomplete gamma function can be implemented by using numerical methods. Consequently, random number can be generated based on (8).

\subsection{Expansion of the GEMW Density Function}

Consider the series $-\log (1-y)=\sum_{0}^{\infty} \frac{y^{i+1}}{i+1}$, where $0<y<1$, and $\mathrm{y}=e^{-\left(\frac{x}{\lambda}\right)^{k} e^{\beta x}}$. The GEMW distribution can be written as

$$
g(x)=\frac{\alpha^{\delta}}{\lambda \Gamma(\delta)}(k+\beta x) e^{\beta x} y\left(\frac{x}{\lambda}\right)^{k-1}\left[\sum_{i=0}^{\infty} \frac{y^{i+1}}{i+1}\right]^{\delta-1} \sum_{r=0}^{\infty}\left(\begin{array}{c}
\alpha-1 \\
r
\end{array}\right)(-1)^{r} y^{r} .
$$

Note that:

$$
\begin{aligned}
\left(\sum_{i=0}^{\infty} \frac{y^{i+1}}{i+1}\right)^{\delta-1} & =\left[y+y^{2} \sum_{s=0}^{\infty} \frac{y^{s}}{s+2}\right]^{\delta-1} \\
& =y^{\delta-1} \sum_{m=0}^{\infty}\left[\left(\begin{array}{c}
\delta-1 \\
m
\end{array}\right) y^{m}\left(\sum_{s=0}^{\infty} \frac{y^{s}}{s+2}\right)^{m}\right] .
\end{aligned}
$$

Next, let $a_{s}=\frac{1}{s+2}$, then $\left(\sum_{s=0}^{\infty} a_{s} y^{s}\right)^{m}=\sum_{s=0}^{\infty} b_{s},{ }_{m} y^{s}$ where

$$
b_{s, m}=\left(s a_{0}\right)^{-1} \sum_{l=1}^{s}[m(l+1)-s] a_{l} b_{s-l, m}
$$

ad $b_{0, m}=a_{0}^{m}$. Now, we can write the GEMW pdf as 


$$
g(x)=\frac{\alpha^{\delta}}{\Gamma(\delta)} \sum_{m=0}^{\infty} \sum_{s, r=0}^{\infty}(-1)^{r}\left(\begin{array}{c}
\delta-1 \\
m
\end{array}\right)\left(\begin{array}{c}
\alpha-1 \\
r
\end{array}\right) \frac{b_{s, m}}{r+m+s+\delta} f_{M W}(x),
$$

where $f_{M W}(x)=f\left(x ; k, \beta, \lambda_{M W}\right)$ denotes the pdf of the modified Weibull distribution (Lai et al., 2003) with $\lambda_{M W}=\frac{\lambda}{(r+m+s+\delta)^{\frac{1}{k}}}>0$. To simplify the notation, one can define $V=$ $\left\{(m, s, r) \in \boldsymbol{Z}_{+}^{3}\right\}$ as an index set and the weights $\mathrm{w}_{v}=\frac{(-1)^{r} \alpha^{\delta}}{\Gamma(\delta)}\left(\begin{array}{c}\delta-1 \\ m\end{array}\right)\left(\begin{array}{c}\alpha-1 \\ r\end{array}\right) \frac{b_{s, m}}{r+m+s+\delta}$, for $v \in$ $V$. Therefore, the GEMW pdf can be written as

$$
g(x)=\sum_{v \in V} w_{v} f_{M W}(x) .
$$

Equation (13) shows that the GEMW density is indeed a linear combination of modified Weibull distribution. Hence, most of its mathematical properties can be immediately obtained from those of the modified Weibull distribution. For the convergence of equations (12) and (13), as well elsewhere in this paper, note that for $>0$;

$$
[-\log (1-y)]^{\delta-1}=\left[y\left(1+y \sum_{s=0}^{\infty} \frac{y^{s}}{s+2}\right)\right]^{\delta-1}
$$

so that

$$
\left[1+y \sum_{k=0}^{\infty} \frac{y^{k}}{k+2}\right]^{\delta-1}=\sum_{k=0}^{\infty}\left(\begin{array}{c}
\delta-1 \\
k
\end{array}\right) y^{k}\left(\sum_{s=0}^{\infty} \frac{y^{s}}{s+2}\right)^{k}
$$

is convergent if and only if $0<\left(y \sum_{k=0}^{\infty} \frac{y^{k}}{k+2}\right)^{k}<1 \forall y \in(0,1)$, since $0<\mathrm{y}=e^{-\left(\frac{x}{\lambda}\right)^{k} e^{\beta x}}<$ $1, x>0, \lambda, k>0$, and $\beta \geq 0$. Now , $0<y \sum_{k=0}^{\infty} \frac{y^{k}}{k+2}=-\frac{\log (1-\mathrm{y})}{\mathrm{y}}-1$, so we must have $0<-\frac{\log (1-\mathrm{y})}{\mathrm{y}}-1<1$. This leads to $1-\mathrm{y}>\exp (-2 \mathrm{y})$, and on the other hand $\exp (-\mathrm{y})=\sum_{k=0}^{\infty} \frac{(-1)^{k} y^{k}}{k !}>1-\mathrm{y}$. Thus, we have the system of inequalities $1-\mathrm{y}>$ $\exp (-2 \mathrm{y})$ and $\exp (-\mathrm{y})>1-\mathrm{y}$, which is statisfied $\forall \mathrm{y} \in(0,0.7968)$.

\subsection{Some Sub-models}

In this section, some sub-models of the GEMW distribution are presented. The GEMW distribution contains several special sub-models that are well known distributions. When $\beta=$ 0 , we obtain

$$
g(x)=\frac{k \alpha}{\lambda \Gamma(\delta)}\left(-\log \left[1-e^{-\left(\frac{x}{\lambda}\right)^{k}}\right]^{\alpha}\right)^{\delta-1}\left(\frac{x}{\lambda}\right)^{k-1} e^{-\left(\frac{x}{\lambda}\right)^{k}}\left[1-e^{-\left(\frac{x}{\lambda}\right)^{k}}\right]^{\alpha-1},
$$

which is the gamma-exponentiated Weibull (GEW) distribution, given by Pinho et al. (2012). The GEW distribution has several special cases as its sub-models. For instance, $=1$ leads to the exponentiated Weibull (EW) distribution (Gupta and Kundu, 2001), with a pdf: 


$$
g(x)=\frac{k \alpha}{\lambda}\left(\frac{x}{\lambda}\right)^{k-1} e^{-\left(\frac{x}{\lambda}\right)^{k}}\left[1-e^{-\left(\frac{x}{\lambda}\right)^{k}}\right]^{\alpha-1} .
$$

When $\alpha=k=1$, EW distribution has the exponential distribution (Gupta and Kundu, 2001) as a sub-model with a pdf

$$
g(x)=\frac{1}{\lambda} e^{-\left(\frac{x}{\lambda}\right)}
$$

By setting $k=1$; EW distribution leads to the exponentiated exponential (EE) with a pdf

$$
g(x)=\frac{\alpha}{\lambda} e^{-\frac{x}{\lambda}}\left(1-e^{-\frac{x}{\lambda}}\right)^{\alpha-1} .
$$

Let $\alpha=1$, from EW distribution we can also obtain Weibull distribution (Weibull, 1951) whose pdf is given by

$$
g(x)=\frac{k}{\lambda}\left(\frac{x}{\lambda}\right)^{k-1} e^{-\left(\frac{x}{\lambda}\right)^{k}} .
$$

When $\beta=0, \alpha=1$ and $\beta=0, \alpha=k=1$, the gamma-Weibull (GW) (Pinho et al., 2012) with a pdf

$$
g(x)=\frac{k}{\lambda \Gamma(\delta)}\left(\frac{x}{\lambda}\right)^{k-1} e^{-\left(\frac{x}{\lambda}\right)^{k}}\left(-\log \left[1-e^{-\left(\frac{x}{\lambda}\right)^{k}}\right]\right)^{\delta-1}
$$

and gamma-exponential (GE) (Pinho et al., 2012) distributions with a pdf

$$
g(x)=\frac{1}{\lambda \Gamma(\delta)} e^{-\frac{x}{\lambda}}\left(-\log \left[1-e^{-\frac{x}{\lambda}}\right]\right)^{\delta-1}
$$

are obtained, respectively. The gamma-exponentiated exponential (GEE) distri-bution follows from $\beta=0$ and $k=1$, whose pdf is given by

$$
g(x)=\frac{\alpha}{\lambda \Gamma(\delta)}\left(-\log \left[1-e^{-\frac{x}{\lambda}}\right]^{\alpha}\right)^{\delta-1} e^{-\frac{x}{\lambda}}\left[1-e^{-\frac{x}{\lambda}}\right]^{\alpha-1} .
$$

Moreover, when $\beta=0, k=2$ and $\beta=0, k=2, \alpha=1$ we obtain gamma-exponentiated Rayleigh (GER) (Ristic and Balakrishnan, 2011) distribution with a pdf

$$
g(x)=\frac{2 x \alpha^{\delta}}{\lambda^{2} \Gamma(\delta)} e^{-\left(\frac{x}{\lambda}\right)^{2}}\left[1-e^{-\left(\frac{x}{\lambda}\right)^{2}}\right]^{\alpha-1}\left(-\log \left[1-e^{-\left(\frac{x}{\lambda}\right)^{2}}\right]\right)^{\delta-1}
$$

and gamma-Rayleigh (GR) distribution (Ristic and Balakrishnan, 2011) with a pdf

$$
g(x)=\frac{2 x}{\lambda^{2} \Gamma(\delta)} e^{-\left(\frac{x}{\lambda}\right)^{2}}\left(-\log \left[1-e^{-\left(\frac{x}{\lambda}\right)^{2}}\right]\right)^{\delta-1}
$$

respectively.

When $\beta \neq 0$, by setting $\alpha=\delta=1$, we obtain the modified Weibull distribution (MW) (Lai et al., 2003), whose pdf is given by 


$$
g(x)=\frac{x^{k-1}}{\lambda^{k}}(k+\beta x) e^{-\left(\frac{x}{\lambda}\right)^{k} e^{\beta x}+\beta x}
$$

For $k=0, \alpha=1$ and $k=0, \delta=\alpha=1$, GEMW gives gamma-extreme value (GEV) (Coles, 2001) and extreme value (EV) (Coles, 2001) distributions, with pdfs

$$
g(x)=\frac{\beta}{\Gamma(\delta)} e^{-e^{\beta x}+\beta x}\left(-\log \left[1-e^{-e^{\beta x}}\right]\right)^{\delta-1}
$$

and

$$
g(x)=\beta e^{-e^{\beta x}+\beta x}
$$

respectively. One can also obtain gamma-modified Weibull (GMW) distribution by letting $\alpha=1$ in GEMW distribution, the corresponding pdf of GMW is:

$$
g(x)=\frac{1}{\lambda \Gamma(\delta)}\left(-\log \left[1-e^{-\left(\frac{x}{\lambda}\right)^{k} e^{\beta x}}\right]\right)^{\delta-1}(k+\beta x)\left(\frac{x}{\lambda}\right)^{k-1} e^{-\left(\frac{x}{\lambda}\right)^{k} e^{\beta x}+\beta x}
$$

which is a new distribution.

\section{Moments, moment generating and characteristic functions}

In this section, the moments, moment generating function and characteristic function of the GEMW distribution are presented. Let $Z$ be a random variable with a density function $g(z)$, as in equation (6) and $\mathrm{X}$ be a random variable with modified Weibull density function $f_{M W}(x)$.

\subsection{Moments}

As mention earlier in section 2.3, the GEMW distribution is a linear combination of modified Weibull distribution. Recall the general results for the beta-modi ed Weibull (BMW) distribution given by Nadarajah et al. (2011). Let $Y \sim B M W(a, b, \alpha, \gamma, \lambda)$ with pdf

$$
f(y)=\frac{\alpha y^{\gamma-1}(\gamma+\lambda y) e^{\lambda y}}{B(a, b)}\left[1-e^{-\alpha y^{\gamma} e^{\lambda y}}\right]^{a-1} e^{-b \alpha y^{\gamma} e^{\lambda y}}, y>0
$$

where $a, b, \alpha>0$ and $\gamma, \lambda \geq 0$. Note that $f_{M W}(x)$ is a sub-model of BMW distribution (Silva et al., 2010) with the parameters:

$$
a=b=1, \gamma=k, \lambda=\beta, \alpha=\frac{r+m+s+\delta}{\lambda^{k}}
$$

Using the moments of BMW distribution, the moments of the MW distribution (Nadarajah et al., 2011) are as follow:

$$
E\left(X^{t}\right)=\sum_{j=0}^{\infty} \omega_{j} I_{1}(j, t)
$$


where $I_{1}(j, t), w_{j}$ and $a_{j}$ given by

$$
\begin{gathered}
I_{1}(j, t)=\sum_{m_{1}=1}^{\infty} \cdots \sum_{m_{t}=1}^{\infty} a_{m_{1}} \cdots a_{m_{t}} \times\left[\frac{r+m+s+\delta}{\lambda^{k}}(1+j)\right]^{-\frac{m_{1}+\cdots+m_{t}}{k}} \\
\times \Gamma\left(\frac{m_{1}+\cdots+m_{t}}{k}+1\right) \\
\omega_{j}=\frac{(-1)^{j}}{\Gamma(1-j)(1+j) j !}
\end{gathered}
$$

and

$$
a_{j}=\frac{(-1)^{j+1} j^{j-2} \beta^{j-1}}{(j-1) ! k^{j-1}},
$$

respectively.

There is also another relatively simpler form of the tth moment by using the Lambert $\mathrm{W}$ (.) function. We can get the following equation for $I_{1}(j, t)$,

$$
I_{1}(j, t)=t(-k)^{t} \sum_{n=1}^{\infty} \frac{(-1)^{n} n^{n-t}(n-1) \cdots(n-t+1)}{n ! k^{n}\left[\frac{r+m+s+\delta}{\lambda^{k}}(1+j)\right]^{\frac{n}{k}}} \Gamma\left(\frac{n}{k}+1\right) .
$$

Substituting equation (18) in (14) gives a representation for moments of the MW (Lai et al., $2003)$ distribution in a relatively concise form with only a doubly infinite series. Thus, the moments of GEMW can be obtained from the moments above. Let $Z \sim \operatorname{GEM} W(\lambda, \delta, \alpha, \beta, k)$, then $E\left(Z^{t}\right)$ can be expressed in terms of the $t^{t h}$ moments of the same baseline MW distribution, which

$$
E\left(Z^{t}\right)=\sum_{v \in V} w_{v} E\left(X^{t}\right)
$$

where $\mathrm{E}(\mathrm{Xt})$ is defined by equation (14), and by substituting $I_{1}(j, t)$ from equation (18), one can also get another form of moments for GEMW distribution.

Conditional expectations are very useful for lifetime models, so it is very important to know $\mathrm{E}\left(X^{t} \mid X>x\right)$, which is given by:

$$
E\left(X^{t} \mid X>x\right)=\frac{\Gamma(\delta)}{\gamma\left\{-\log \left[1-e^{-\left(\frac{x}{\lambda}\right)^{k} e^{\beta x}}\right]^{\alpha}, \delta\right\}} \sum_{j=0}^{\infty} w_{j} I_{2}(j, t),
$$

where

$$
\begin{aligned}
I_{2}(j, t)= & \sum_{m_{1}=1}^{\infty} \cdots \sum_{m_{t}=1}^{\infty} a_{m_{1}} \cdots a_{m_{t}} \times\left[\frac{r+m+s+\delta}{\lambda^{k}}(1+j)\right]^{-\frac{m_{1}+\cdots+m_{t}}{k}} \\
& \times \Gamma\left(\frac{m_{1}+\cdots+m_{t}}{k}+1, \frac{r+m+s+\delta}{\lambda^{k}}(1+j) x\right)
\end{aligned}
$$


and $w_{j}$ and $a_{j}$ are given by (16), (17) and $\Gamma(a, x)=\int_{x}^{\infty} y^{a-1} e^{-y} d y$.

Similarly, a relatively simpler representation for $\mathrm{E}\left(X^{t} \mid X>x\right)$ can be obtained from equation (18),

$$
\begin{aligned}
I_{2}(j, t)= & t(-k)^{t} \beta^{-t} \sum_{n=1}^{\infty} \frac{(-\beta)^{n} n^{n-t}(n-1) \cdots(n-t+1)}{n ! k^{n}\left[\frac{r+m+s+\delta}{\lambda^{k}}(1+j)\right]^{\frac{n}{k}}} \\
& \times \Gamma\left(\frac{n}{k}+1, \frac{r+m+s+\delta}{\lambda^{k}}(1+j) x\right) .
\end{aligned}
$$

\subsection{Moment generating and characteristic functions}

Let $X \sim G E M W(\lambda, \delta, \alpha, \beta, k)$, the moment generating function and characteristic function of $X$ are given by $M(t)=E\left(e^{t x}\right)$ and $\phi(t)=E\left(e^{i t x}\right)$, respectively, where $i=\sqrt{-1}$. Note that $M(t)$ and $\phi(t)$ can be express as $M(t)=\sum_{k=0}^{\infty} \frac{t^{k}}{k !} E\left(X^{k}\right)$ and $\phi(t)=\sum_{k=0}^{\infty} \frac{(i t)^{k}}{k !} E\left(X^{k}\right)$, where $E\left(X^{k}\right)$ is the given by equation (14). Nothing that, $g(x)$ can be expressed as an infinite weighted sum, we have

$$
M(t)=\sum_{v \in V} w_{v} M_{M W}(t)
$$

Where $M_{M W}(t)$ is the moment generating function of the MW distribution, which is,

$$
M_{M W}(t)=-t k \sum_{j=0}^{\infty} \sum_{n=0}^{\infty} \frac{(-1)^{n} \omega_{j}(n \beta-t k)^{n-1}}{n ! k^{n}\left[\frac{r+m+s+\delta}{\lambda^{k}}(1+j)\right]^{\frac{n}{k}}} \Gamma\left(\frac{n}{k}+1\right) .
$$

The corresponding characteristic function is

$$
\phi(t)=\sum_{v \in V} w_{v} \phi_{M W}(t)
$$

with

where $w_{j}$ is given by equation (16).

$$
\phi_{M W}(t)=-i t k \sum_{j=0}^{\infty} \sum_{n=0}^{\infty} \frac{(-1)^{n} \omega_{j}(n \beta-t k)^{n-1}}{n ! k^{n}\left[\frac{r+m+s+\delta}{\lambda^{k}}(1+j)\right]^{\frac{n}{k}}} \Gamma\left(\frac{n}{k}+1\right)
$$

\section{Mean deviations, Bonferroni and Lorenz curves}

In this section, mean deviations about the mean and the median, Bonferroni and Lorenz curves of the GEMW distribution are represented.

\subsection{Mean deviations}

Let $X \sim G E M W(\delta, \alpha, \beta, k, \lambda)$, the mean deviation about the mean and the mean deviation about the median are defined by $\delta_{1}(X)=\int_{0}^{\infty}|x-u| g(x) d x$, and $\delta_{2}(X)=\int_{0}^{\infty} \mid x-$ 
$M \mid g(x) d x$, respectively, where $\mu=\mathrm{E}(\mathrm{X})$ and $M=\operatorname{Median}(X)$ denotes the median. The measures $\delta_{1}(X)$ and $\delta_{2}(X)$ can be calculated using the relationships

$$
\delta_{1}(X)=2 \mu G(\mu)-2 \mu+2 \int_{\mu}^{\infty} x g(x) d x
$$

and

$$
\delta_{2}(X)=2 \int_{M}^{\infty} x g(x) d x-\mu
$$

Recall that $g(x)=\sum_{v \in V} \omega_{u} f_{M W}(x)$, so that $\int_{\mu}^{\infty} x g(x) d x=\sum_{v \in V} \omega_{u} I_{3} \quad$ and $\int_{M}^{\infty} x g(x) d x=\sum_{v \in V} \omega_{u} I_{4}$, where

$$
\begin{aligned}
& I_{3}=\sum_{j=0}^{\infty} \sum_{m=1}^{\infty} \omega_{j} a_{m}\left[\frac{r+m+s+\delta}{\lambda^{k}}(1+j)\right]^{-\frac{m}{k}} \Gamma\left(\frac{m}{k}+1, \frac{r+m+s+\delta}{\lambda^{k}}(1+j) \mu\right), \\
& I_{4}=\sum_{j=0}^{\infty} \sum_{m=1}^{\infty} \omega_{j} a_{m}\left[\frac{r+m+s+\delta}{\lambda^{k}}(1+j)\right]^{-\frac{m}{k}} \Gamma\left(\frac{m}{k}+1, \frac{r+m+s+\delta}{\lambda^{k}}(1+j) M\right),
\end{aligned}
$$

and $\omega_{j}$ and $a_{j}$ are given by equation (16) and (17), respectively. It follows that

$$
\delta_{1}(X)=2 \mu G(\mu)-2 \mu+2 \sum_{v \in V} w_{v} I_{3}, \quad \text { and } \quad \delta_{2}(X)=2 \sum_{v \in V} w_{v} I_{4}-\mu .
$$

\subsection{Bonferroni and Lorenz Curves}

In this section, some inequality measures, namely Bonferroni and Lorenz curves are presented. These quantities have been applied to a wide variety of fields, such as studying of income and property in economics, reliability, demography, insurance and medicine.

For $X \sim G E M W(\delta, \alpha, \beta, k, \lambda)$, they are defined by

$$
B(p)=\frac{1}{p \mu} \int_{0}^{q} x g(x) d x \text { and } L(p)=\frac{1}{\mu} \int_{0}^{q} x g(x) d x,
$$

respectively, where $\mu=E(X)$ and $\mathrm{q}=G^{-1}(p)$ is obtained from equation (8).

Using similar methods in deriving the moments, we can show that

$$
\int_{0}^{q} x g(x) d x=\sum_{v \in V} \sum_{j=0}^{\infty} w_{v} \omega_{j} I_{5}(j)
$$

where

$$
I_{5}(j)=\sum_{m=1}^{\infty} a_{m}\left[\frac{r+m+s+\delta}{\lambda^{k}}(1+j)\right]^{-\frac{m}{k}} \gamma\left(\frac{m}{k}+1,(1+j) q\right),
$$


and $\omega_{j}$ and $a_{j}$ are given by equation (16) and (17), respectively. We can reduce the curves in equation (25) to

$$
B(p)=\frac{1}{p \mu} \sum_{v \in V} \sum_{j=0}^{\infty} w_{v} \omega_{j} I_{5}(j) \text { and } L(p)=\frac{1}{\mu} \sum_{v \in V} \sum_{j=0}^{\infty} w_{v} \omega_{j} I_{5}(j),
$$

respectively.

\section{Order Statistics and Renyi Entropy}

In this section, the distribution of the ith order statistic and Renyi entropy for the GEMW distribution are presented.

\subsection{Order Statistics}

Consider $X_{1}, \ldots . . X_{n}$ i.i.d random variables distributed according to (2). The pdf of the ith order statistic, say $X_{i: n}$, is given by

$$
g_{i: n}(x)=\frac{n ! g(x)}{(i-1) !(n-i) !}[\bar{G}(x)]^{n-i}[1-\bar{G}(x)]^{i-1} .
$$

Using the binomial theorem, the pdf of $i^{\text {th }}$ order statistic can be written as

$$
g_{i: n}(x)=\frac{n ! g(x)}{(i-1) !(n-i) !} \sum_{j=0}^{i-1}(-1)^{j}\left(\begin{array}{c}
i-1 \\
j
\end{array}\right)\left\{\frac{\gamma[-\log F(x), \delta]}{\Gamma(\delta)}\right\}^{n+j-i} .
$$

Applying the power series (see Gradshteyn and Ryzhik, 2000)

$$
\gamma(x, \delta)=\sum_{m=0}^{\infty} \frac{(-1)^{m} x^{m+\delta}}{(m+\delta) m !}
$$

we have

$$
\begin{aligned}
g_{i: n}(x) & =\frac{n ! g(x)}{(i-1) !(n-i) !} \sum_{j=0}^{i-1}\left(\begin{array}{c}
i-1 \\
j
\end{array}\right) \frac{(-1)^{j}}{[\Gamma(\delta)]^{n+j-i}}[-\log F(x)]^{\delta(n+j-i)} \\
& \times\left\{\sum_{m=0}^{\infty} \frac{(-1)^{m}[-\log F(x)]^{m}}{(m+\delta) m !}\right\}^{n+j-i} .
\end{aligned}
$$

Let $c_{m}=\frac{(-1)^{m}}{m !(m+\delta)}$ and use the result on a power series raised to a positive integer, as in section 2.3 , to write

$$
\left\{\sum_{m=0}^{\infty} c_{m}[-\log F(x)]^{m}\right\}^{n+j-i}=\sum_{m=0}^{\infty} d_{m, n+j-i}[-\log F(x)]^{m},
$$


where $\quad d_{0, n+j-i}=c_{0}^{(n+j-i)} \quad$ and $\quad d_{m, n+j-i}=\left(m c_{0}\right)^{-1} \sum_{l=1}^{m}[(n+j-i) l-m+$ $l] c_{l} d_{m-l, n+j-i}$. Replacing $g(x)$ by the right hand side of (2), we obtain

$$
\begin{aligned}
g_{i: n}(x) & =\frac{n !}{(i-1) !(n-i) !}\left\{\sum_{j=0}^{i-1} \sum_{m=0}^{\infty}\left(\begin{array}{c}
i-1 \\
j
\end{array}\right)\right. \\
& \left.\times \frac{(-1)^{j} d_{m, n+j-i}}{[\Gamma(\delta)]^{n+j-i+1}} \Gamma(\delta(n+j-i+1)+m) g_{\delta(n+j-i+1)+m}(x)\right\},
\end{aligned}
$$

where $g_{\delta(n+j-i+1)+m}(x)$ denotes the GEMW with $\delta_{*}=\delta(n+j-i+1)+m>0$. Consequently, $g_{i: n}(x)$ is a linear combination of modified Weibull densities. This is very useful result since we can derive properties of the order statistics of the GEMW distribution from those of modified Weibull distribution. For instance, we can obtain

$$
\begin{aligned}
E\left(X_{i: n}^{t}\right) & =\frac{n !}{(i-1) !(n-i) !} \sum_{v \in V} \sum_{j=0}^{i-1} \sum_{m=0}^{\infty} \sum_{l=0}^{\infty}\left(\begin{array}{c}
i-1 \\
j
\end{array}\right) \frac{(-1)^{j} \omega_{v} d_{m, n+j-i}}{[\Gamma(\delta)]^{n+j-i+1}} \\
& \times \Gamma(\delta(n+j-i+1)+m) I_{1}(l, t),
\end{aligned}
$$

where $I_{1}(l, t)$ is given by equation (15). These moments are used in areas such as quality control, reliability and insurance, for prediction of future failure times from a set of past failures.

\subsection{Renyi Entropy}

Renyi entropy is defined by

$$
I_{R}(\nu)=(1-\nu)^{-1} \log \left[\int_{-\infty}^{\infty} g^{\nu}(x) d x\right],
$$

where $v>0$ and $v \neq 1$. Raising equation (6) to the power and using the similar expansion in section 2 , we obtain

$$
\begin{aligned}
{[g(x)]^{\nu} } & =\frac{\alpha^{\delta \nu}}{\lambda^{\mu} \Gamma^{\nu}(\delta)} \sum_{m, s, r, l, i=0}^{\infty}\left(\begin{array}{c}
(\delta-1) \nu \\
m
\end{array}\right)\left(\begin{array}{c}
(\alpha-1) \nu \\
r
\end{array}\right)\left(\begin{array}{c}
\nu-1 \\
l
\end{array}\right) \\
& \times(-1)^{r} b_{s, m} \beta^{l+i} k^{\nu-l-1} \frac{(\nu-1)^{i}}{i !} \frac{x^{(\nu-1)(k-1)+l+i}}{\delta \nu+m+s+r} \\
& \times \frac{1}{\lambda_{* *}}\left(\frac{x}{\lambda_{* *}}\right)^{k-1} e^{\beta x}(k+\beta x) e^{\left(\frac{x}{\lambda_{* *}}\right)^{k} e^{\beta x}},
\end{aligned}
$$

where $\lambda_{* *}=\frac{\lambda}{(\delta v+m+s+r)^{1 / k}}>0$ and $b_{s, m}$ is given by equation (11). Consequently Renyi entropy for the GEMW distribution reduces to: 


$$
\begin{aligned}
I_{R}(\nu) & =(1-\nu)^{-1} \log \left[\frac{\alpha^{\delta \nu}}{\lambda^{\mu} \Gamma^{\nu}(\delta)} \sum_{m, s, r, l, i, j=0}^{\infty} \sum_{n=1}^{\infty}\left(\begin{array}{c}
(\delta-1) \nu \\
m
\end{array}\right)\left(\begin{array}{c}
(\alpha-1) \nu \\
r
\end{array}\right)\left(\begin{array}{c}
\nu-1 \\
l
\end{array}\right)\right. \\
& \times(-1)^{r} \omega_{j} b_{s, m}[(\nu-1)(k-1)+l+i] \beta^{l+i} k^{\nu-l-1} \Gamma\left(\frac{n}{k}+1\right) \\
& \left.\times \frac{(\nu-1)^{i}(-k)^{[(\nu-1)(k-1)+l+i]}}{(\delta \nu+m+s+r) i !} \frac{(-1)^{n} n^{n-[(\nu-1)(k-1)+l+i]}(n-1) \cdots(n-t+1)}{n ! k^{n}\left[\frac{r+m+s+\delta \nu}{\lambda^{k}}(1+j)\right]^{\frac{n}{k}}}\right]
\end{aligned}
$$

where $\omega_{j}$ and $a_{j}$ are given by equation (16) and (17), and $\Gamma(\mathrm{a})=\int_{0}^{\infty} y^{a-1} e^{-y} d y$.

\section{Estimation of Parameters}

Let $X \sim G E M W(\delta, \alpha, \beta, k, \lambda)$ and $\Delta=(\delta, \alpha, \beta, k, \lambda)^{T}$ be the parameter vector. The loglikelihood for a single observation $x$ of $\mathrm{X}$ is given by

$$
\begin{aligned}
\ell=\ell(\boldsymbol{\Delta})= & (\delta-1) \log \left(-\alpha \log \left(1-e^{-\left(\frac{x}{\lambda}\right)^{k} e^{\beta x}}\right)\right)-\log [\Gamma(\delta)]+\log (k+\beta x) \\
& +\log (\alpha)-k \log (\lambda)+(k-1) \log (x)+\beta x \\
& -\left(\frac{x}{\lambda}\right)^{k} e^{\beta x}+(\alpha-1) \log \left(1-e^{-\left(\frac{x}{\lambda}\right)^{k} e^{\beta x}}\right) .
\end{aligned}
$$

The first derivative of the log-likelihood function with respect to the parameters $\Delta=(\delta, \alpha, \beta, k, \lambda)^{T}$ are given by

$$
\begin{aligned}
& \frac{\partial \ell}{\partial \alpha}=\frac{\delta}{\alpha}+\log \left[1-e^{-\left(\frac{x}{\lambda}\right)^{k} e^{\beta x}}\right] \\
& \frac{\partial \ell}{\partial \beta}=\frac{(\delta-1) x\left(\frac{x}{\lambda}\right)^{k} e^{-\left(\frac{x}{\lambda}\right)^{k} e^{\beta x}+\beta x}}{\left(\log \left(1-e^{-\left(\frac{x}{\lambda}\right)^{k} e^{\beta x}}\right)\right)\left(1-e^{-\left(\frac{x}{\lambda}\right)^{k} e^{\beta x}}\right)}+\frac{x}{k+\beta x} \\
& +x-\left(\frac{x}{\lambda}\right)^{k} e^{\beta x} x+\frac{x(\alpha-1)\left(\frac{x}{\lambda}\right)^{k} e^{\beta x} e^{-\left(\frac{x}{\lambda}\right)^{k} e^{\beta x}+\beta x}}{1-e^{-\left(\frac{x}{\lambda}\right)^{k} e^{\beta x}}}, \\
& \frac{\partial \ell}{\partial \lambda}=\frac{(1-\delta) k x^{k} \lambda^{-k-1} e^{-\left(\frac{x}{\lambda}\right)^{k} e^{\beta x}+\beta x}}{\left(\log \left(1-e^{-\left(\frac{x}{\lambda}\right)^{k} e^{\beta x}}\right)\right)\left(1-e^{-\left(\frac{x}{\lambda}\right)^{k} e^{\beta x}}\right)}-\frac{k}{\lambda} \\
& +\frac{k x^{k} e^{\beta x}}{\lambda^{k+1}}+\frac{k(\alpha-1) x^{k} e^{\beta x-\left(\frac{x}{\lambda}\right)^{k} e^{\beta x}}}{\lambda^{k+1}\left(1-e^{-\left(\frac{x}{\lambda}\right)^{k} e^{\beta x}}\right)}, \\
& \frac{\partial \ell}{\partial k}=\frac{(\delta-1)\left(\frac{x}{\lambda}\right)^{k} \log \left(\frac{x}{\lambda}\right)}{\left(\log \left(1-e^{-\left(\frac{x}{\lambda}\right)^{k} e^{\beta x}}\right)\right)\left(1-e^{-\left(\frac{x}{\lambda}\right)^{k} e^{\beta x}}\right)}+\frac{1}{k+\beta x}-\log (\lambda)+\log (x) \\
& -\log \left(\frac{x}{\lambda}\right)\left(\frac{x}{\lambda}\right)^{k}\left[\log \left(\frac{x}{\lambda}\right)\right] e^{\beta x}+\frac{(\alpha-1)\left(\frac{x}{\lambda}\right)^{k} \log \left(\frac{x}{\lambda}\right) e^{-\left(\frac{x}{\lambda}\right)^{k} e^{\beta x}+\beta x}}{1-e^{-\left(\frac{x}{\lambda}\right)^{k} e^{\beta x}}}
\end{aligned}
$$

and 


$$
\frac{\partial \ell}{\partial \delta}=\log \left(-\alpha \log \left(1-e^{-\left(\frac{x}{\lambda}\right)^{k} e^{\beta x}}\right)\right)-\frac{\Gamma^{\prime}(\delta)}{\Gamma(\delta)} .
$$

The total log-likelihood function based on a random sample of $n$ observations: $\left\{x_{1}, x_{2}, \ldots \ldots x_{n}\right\}$ drawn from the GEMW distribution is given by $\ell^{*}=L(\Delta)=$ $\sum_{i=1}^{n} \ell_{i}(\Delta)$, where $\ell_{i}(\Delta), i=1,2, \ldots \ldots, \mathrm{n}$ is given by equation (30). The equations obtained by setting the above partial derivatives to zero are not in closed form and the values of the parameters $\alpha, \beta, \theta, \lambda, \delta$ must be found by using iterative methods. The maximum likelihood estimates of the parameters, denoted by $\widehat{\Delta}$ is obtained by solving the nonlinear equation $\left(\frac{\partial \ell^{*}}{\partial \alpha}, \frac{\partial \ell^{*}}{\partial \beta}, \frac{\partial \ell^{*}}{\partial k}, \frac{\partial \ell^{*}}{\partial \lambda}, \frac{\partial \ell^{*}}{\partial \delta}\right)^{\mathrm{T}}=0$, using a numerical method such as Newton-Raphson procedure.

We maximize the likelihood function using NLmixed in SAS as well as the function nlm in R (2011). These functions were applied and executed for wide range of initial values. This process often results or lead to more than one maximum, however, in these cases, we take the MLEs corresponding to the largest value of the maxima. In a few cases, no maximum was identified for the selected initial values. In these cases, a new initial value was tried in order to obtain a maximum.

The issues dealing with the existence and uniqueness of the MLEs are theo-retical interest and has been studied by several authors for di erent distributions including Seregin (2010), Santos Silva and Tenreyro (2010), Zhou (2009), and Xia et al. (2009). We hope to investigate this problem or issue for the GEMW distribution in the future.

Let $\widehat{\Delta}=(\widehat{\alpha}, \widehat{\beta}, \widehat{\mathrm{k}}, \hat{\lambda}, \widehat{\delta})$ be the maximum likelihood estimate of $\Delta=(\alpha, \beta, \mathrm{k}, \lambda, \delta)$ Under the usual regularity conditions and that the parameters are in the inte-rior of the parameter space, but not on the boundary, (Ferguson, 1996) we have:

$\sqrt{n}(\widehat{\Delta}-\Delta) \stackrel{d}{\rightarrow} N_{5}\left(\underline{0}, I^{-1}(\Delta)\right)$, where $I(\Delta)$ is the expected Fisher information matrix. The asymptotic behavior is still valid if $I(\Delta)$ is replaced by the observed information matrix evaluated at $\widehat{\Delta}$, that is $\mathrm{J}(\widehat{\Delta})$. Elements of the observed information matrix are given in the Appendix. The multivariate normal distribution $N_{5}\left(\underline{0}, J(\hat{\Delta})^{-1}\right)$, where the mean vector $\underline{0}=(0$, $0,0,0,0) \mathrm{T}$, can be used to construct confidence intervals and confidence regions for the individual model parameters and for the survival and hazard rate functions. That is, the approximate $100(1-\eta) \%$ two-sided confidence intervals for $\alpha, \beta, \mathrm{k} \lambda$, and $\delta$ are given by:

$$
\widehat{\alpha} \pm Z_{\frac{n}{2}} \sqrt{I_{\alpha \alpha}^{-1}(\hat{\boldsymbol{\Delta}})}, \quad \widehat{\beta} \pm Z_{\frac{n}{2}} \sqrt{I_{\beta \beta}^{-1}(\hat{\boldsymbol{\Delta}})}, \quad \hat{k} \pm Z_{\frac{n}{2}} \sqrt{I_{k k}^{-1}(\hat{\boldsymbol{\Delta}})}, \quad \hat{\lambda} \pm Z_{\frac{n}{2}} \sqrt{I_{\lambda \lambda}^{-1}(\hat{\boldsymbol{\Delta}})},
$$

and $\hat{\delta} \pm Z_{\frac{\eta}{2}} \sqrt{I_{\delta \delta}^{-1}(\hat{\Delta})}$, respectively, where $I_{\alpha \alpha}^{-1}(\widehat{\Delta}), I_{\beta \beta}^{-1}(\widehat{\Delta}), I_{k k}^{-1}(\widehat{\Delta}), I_{\lambda \lambda}^{-1}(\widehat{\Delta})$ and $I_{\delta \delta}^{-1}(\widehat{\Delta})$ are the diagonal elements of $I_{n}^{-1}(\widehat{\Delta})=(n I(\widehat{\Delta}))^{-1}$, and $Z_{\frac{\eta}{2}}$ is the upper $\frac{\eta^{t h}}{2}$ percentile od a standard normal distribution.

The maximum likelihood estimates (MLEs) of the GEMW parameters $\alpha, \beta, \theta, \lambda$, and $\delta$ are computed by maximizing the objective function via the subroutine NLmixed in SAS. The estimated values of the parameters (standard error in parenthesis), -2log-likelihood statistic, Akaike Information Criterion, AIC $=2 p-2 \ln (\mathrm{L})$, Bayesian Information Criterion, BIC $=p$ $\ln (\mathrm{n})-2 \ln (\mathrm{L})$, and Consistent Akaike Information Criterion, AICC $=\mathrm{AIC}+2 \frac{p(p+1)}{n-p-1}$, where $\mathrm{L}=\mathrm{L}(\hat{\Delta})$ is the value of the likelihood function evaluated at the parameter estimates, $\mathrm{n}$ is the 
number of observations, and $\mathrm{p}$ is the number of estimated parameters, and KolmogorovSmirnov (KS) statistic are presented in Tables 2 and 3.

In order to compare the models, we use the criteria stated above. Note that for the value of the log-likelihood function at its maximum $(\mathrm{L}(\widehat{\Delta}))$, larger value is good and preferred, and for the Kolmogorov-Smirnov test statistic (K-S), smaller value is preferred. GEMW distribution is fitted to the data sets and these fits are compared to the fits using the Exponential, Weibull, EE, GE, GEE, GW, EW, MW, GEW and GMW distributions.

We can use the likelihood ratio (LR) test to compare the fit of the GEMW distribution with its sub-models for a given data set. For example, to test $\lambda=\delta=1$, the LR statistic is $\omega=2[\ln (L(\hat{\alpha}, \hat{\beta}, \hat{k} . \hat{\lambda} . \hat{\delta}))-\ln (L(\tilde{\alpha}, \tilde{\beta}, \tilde{k}, 1,1))]$, where $\widehat{\alpha}, \hat{\beta}, \hat{k} . \hat{\lambda}$ and $\hat{\delta}$, are the unrestricted estimates, and $\tilde{\alpha}, \tilde{\beta}$, and $\tilde{k}$ are the restricted estimates. The LR test rejects the null hypothesis if $\omega>\chi_{\epsilon}^{2}$, where $\chi_{\epsilon}^{2}$ denote the upper $100 \mathrm{~s} \%$ point of the $\chi^{2}$ distribution with 2 degrees of freedom.

\section{Applications}

In this section, we present two examples to illustrate the flexibility of the GEMW distribution and its sub-models for data modeling.

The first data consists of the lifetimes of $n=50$ devices given by Aarset (1987). It is known to have a bathtub-shaped hazard function thus been widely studied. The dataset are: 0.10 .21 .01 .01 .01 .01 .02 .03 .06 .07 .011 .012 .018 .018 .018 .018 .018 .021 .032 .036 .0 40.045 .046 .047 .050 .055 .060 .063 .063 .067 .067 .067 .067 .072 .075 .079 .082 .082 .0 83.084 .084 .084 .085 .085 .085 .085 .085 .086 .086 .0 .

The second data gives failure and running times of a sample of $n=30$ devices given by Meeker and Escobar (1998). This data has a bathtub shaped hazard function and are given by: 2101323232830658088106143147173181212245247261266275293300300 300300300300300300 .

Some descriptive statistics of these two data sets are given in Table 1.

Table 1: GEMW Descriptive Statistics of Application Data Sets

\begin{tabular}{cccccccc}
\hline Data & $\mathrm{n}$ & Mean & Media & Minimu & Maximu & Varianc & SD \\
\hline Aarset & 50 & 45.686 & 48.5 & 0.1 & 86.0 & 1078.2 & 32.8352 \\
Meeke & 30 & 177.03 & 196.5 & 2 & 300 & 13223 & 114.991 \\
\hline
\end{tabular}

Estimates of the parameters of GEMW distribution (standard error in parentheses), Akaike Information Criterion (AIC), Consistent Akaike Information Criterion (AICC), Bayesian Information Criterion (BIC) and Kolmogorov-Smirnov (KS) statistic are given in Table 2 for the first data set and in Table 3 for the second data set. The estimated covariance matrix for the GEMW distribution (Aarset Data) is given by

$$
\left[\begin{array}{ccccl}
208.87 & -40.6120 & 0.1575 & 3.3190 & 2.3599 \\
-40.6120 & 0.08240 & -0.00033 & -0.00641 & -0.00453 \\
0.1575 & -0.00033 & 0.000237 & 0.001232 & -0.0003 \\
3.3190 & -0.00641 & 0.001232 & 0.01139 & -0.0006 \\
2.3599 & -0.00453 & -0.00003 & -0.0006 & 0.000294
\end{array}\right]
$$


The 95\% asymptotic confidence intervals for the GEMW model (Aarset Data) parameters are: $\alpha \in(0.01279,0.07463), \beta \in(0.05552,0.1244), \delta \in(0.1628,0.5915), k \in(3.9043,5.0574)$, and $\lambda \in(317.7468,374.2732)$, respectively. 
Table 2: GEMW Estimation for Aarset data

\begin{tabular}{|c|c|c|c|c|c|c|c|c|c|c|c|}
\hline Model & $a$ & $\beta$ & $\lambda$ & k & $\delta$ & -2Log Likelihood & AIC & $\mathrm{BIC}$ & AICC & $\mathrm{KS}$ & SS \\
\hline \multirow[t]{2}{*}{ Exponential } & 1 & 0 & 45.6858 & 1 & 1 & 482.2 & 484.2 & 486.1 & 484.3 & 0.1911 & 0.5190 \\
\hline & - & & $(6.4609)$ & - & - & & & & & & \\
\hline \multirow[t]{2}{*}{ Weibull } & 1 & 0 & 44.9125 & 0.9490 & 1 & 482.0 & 486.0 & 489.8 & 486.3 & 0.1928 & 0.5289 \\
\hline & - & & $(6.9451)$ & (0.1196) & & & & & & & \\
\hline \multirow[t]{2}{*}{$\mathrm{EE}$} & 0.7798 & 0 & 53.4739 & 1 & 1 & 480.0 & 484.0 & 487.8 & 484.2 & 0.2042 & 0.5634 \\
\hline & $(10.3680)$ & & $(0.1351)$ & - & 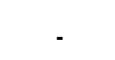 & & & & & & \\
\hline \multirow[t]{2}{*}{ GE } & 1 & 0 & 70.2848 & 1 & 1.3798 & 481.6 & 485.6 & 489.4 & 485.8 & 0.1981 & 0.5469 \\
\hline & - & & $(40.4183)$ & - & $(0.5253)$ & & & & & & \\
\hline \multirow[t]{2}{*}{ GEE } & 0.3148 & 0 & 7.0083 & 1 & 0.1403 & 477.2 & 483.2 & 488.9 & 483.7 & 0.1911 & 0.4920 \\
\hline & $(0.2004)$ & - & $(7.8173)$ & - & $(0.1521)$ & & & & & & \\
\hline \multirow[t]{2}{*}{ GW } & 1 & 0 & 83.0358 & 1.0597 & 1.5605 & 481.5 & 487.5 & 493.2 & 488.0 & 0.1974 & 0.5492 \\
\hline & - & - & $(12.9968)$ & $(0.1425)$ & $(0.3661)$ & & & & & & \\
\hline \multirow[t]{2}{*}{ EW } & 0.1275 & 0 & 91.0063 & 5.4117 & 1 & 457.0 & 463.0 & 468.7 & 465.3 & 0.2092 & 0.5679 \\
\hline & $(0.01843)$ & - & $(6.5188)$ & $(0.1637)$ & - & & & & & & \\
\hline \multirow[t]{2}{*}{ MW } & 1 & 0.02332 & 2487.20 & 0.3548 & 1 & 454.3 & 460.3 & 466.0 & 460.8 & 0.1337 & 0.2662 \\
\hline & - & $(0.002633)$ & $(1.107)$ & $(0.05343)$ & & & & & & & \\
\hline \multirow[t]{2}{*}{ GEW } & 0.08204 & 0 & 82.8604 & 5.5040 & 0.6182 & 451.8 & 458.7 & 466.3 & 459.6 & 0.1353 & 0.2248 \\
\hline & $(0.00714)$ & & $(20.5673)$ & $(0.1289)$ & $(0.0145)$ & & & & & & \\
\hline \multirow[t]{2}{*}{ GMW } & 1 & 0.02165 & 1231.0 & 0.3955 & 1.0017 & 454.5 & 462.5 & 470.1 & 463.4 & 0.1418 & 0.2773 \\
\hline & - & $(0.003056)$ & $(0.000125)$ & $(0.1584)$ & $(0.4751)$ & & & & & & \\
\hline \multirow[t]{2}{*}{ GEMW } & 0.04371 & 0.08996 & 346.01 & 4.4809 & 0.3771 & 434.3 & 444.3 & 453.9 & 445.7 & 0.1292 & 0.1497 \\
\hline & $(0.01539)$ & $(0.01714)$ & $(14.452)$ & $(0.2870)$ & $(0.1067)$ & & & & & & \\
\hline
\end{tabular}


Table 3: GEMW Estimation for Meeker and Escobar data

\begin{tabular}{|c|c|c|c|c|c|c|c|c|c|c|c|}
\hline Model & a. & $\beta$ & $\lambda$ & $\mathrm{k}$ & $\delta$ & -2 Log Likelihood & AIC & BIC & AICC & KS & SS \\
\hline \multirow[t]{2}{*}{ Exponential } & 1 & 0 & 172.75 & 1 & 1 & 356.8 & 358.8 & 360.2 & 359.0 & 0.2061 & 0.3184 \\
\hline & . & - & (32.0784) & - & - & & & & & & \\
\hline \multirow[t]{2}{*}{ Weibull } & 1 & 0 & 182.77 & 1.2359 & 1 & 355.3 & 359.3 & 362.0 & 359.7 & 0.2105 & 0.2937 \\
\hline & - & - & (28.5726) & $(0.2023)$ & - & & & & & & \\
\hline \multirow[t]{2}{*}{$\mathrm{EE}$} & 1.1280 & 0 & 160.55 & 1 & 1 & 356.6 & 360.6 & 363.3 & 361.0 & 0.2067 & 0.3055 \\
\hline & $(0.2710)$ & - & $(36.5670)$ & - & - & & & & & & \\
\hline \multirow[t]{2}{*}{ GE } & 1 & 0 & 119.99 & 1 & 0.7315 & 356.5 & 360.5 & 363.2 & 361.0 & 0.2047 & 0.3015 \\
\hline & - & - & (40.4183) & - & $(0.5253)$ & & & & & & \\
\hline \multirow[t]{2}{*}{ GEE } & 1.1370 & 0 & 170.00 & 1 & 1.0471 & 356.6 & 362.6 & 366.7 & 363.5 & 0.2042 & 0.2995 \\
\hline & $(0.2004)$ & - & $(7.8173)$ & - & $(0.1521)$ & & & & & & \\
\hline \multirow[t]{2}{*}{ GW } & 1 & 0 & 330.00 & 1.4291 & 1.7402 & 354.9 & 360.9 & 365.0 & 361.9 & 0.2145 & 0.3033 \\
\hline & - & - & $(6.8530)$ & $(0.4399)$ & $(1.4851)$ & & & & & & \\
\hline \multirow[t]{2}{*}{ EW } & 0.1286 & 0 & 325.36 & 6.8594 & 1 & 341.7 & 347.7 & 351.8 & 348.7 & 0.2199 & 0.2807 \\
\hline & $(0.01843)$ & - & (6.5188) & $(0.1637)$ & - & & & & & & \\
\hline \multirow[t]{2}{*}{ MW } & 1 & 0.006861 & 5055.05 & 0.4662 & 1 & 343.9 & 349.9 & 354.0 & 350.8 & 0.1766 & 0.1989 \\
\hline & - & $(0.001021)$ & $(0.00001)$ & $(0.09065)$ & - & & & & & & \\
\hline \multirow[t]{2}{*}{ GEW } & 0.1025 & 0 & 329.47 & 7.1874 & 0.8469 & 340.9 & 348.9 & 354.3 & 350.5 & 0.1784 & 0.1752 \\
\hline & $(0.04242)$ & $\cdot$ & $(10.3241)$ & $(0.4325)$ & $(0.2146)$ & & & & & & \\
\hline \multirow[t]{2}{*}{ GMW } & 1 & 0.005980 & 1786.00 & 0.5343 & 0.9143 & 344.1 & 352.1 & 357.6 & 353.8 & 0.1860 & 0.2054 \\
\hline & - & $(0.003056)$ & $(0.000125)$ & $(0.1584)$ & $(0.4751)$ & & & & & & \\
\hline \multirow[t]{2}{*}{ GEMW } & 0.04764 & 0.03328 & 1567.01 & 5.5337 & 0.4426 & 330.1 & 340.1 & 346.9 & 342.7 & 0.1587 & 0.1446 \\
\hline & $(0.02313)$ & $(0.002379)$ & $(0.000066)$ & (0.1088) & $(0.1846)$ & & & & & & \\
\hline
\end{tabular}



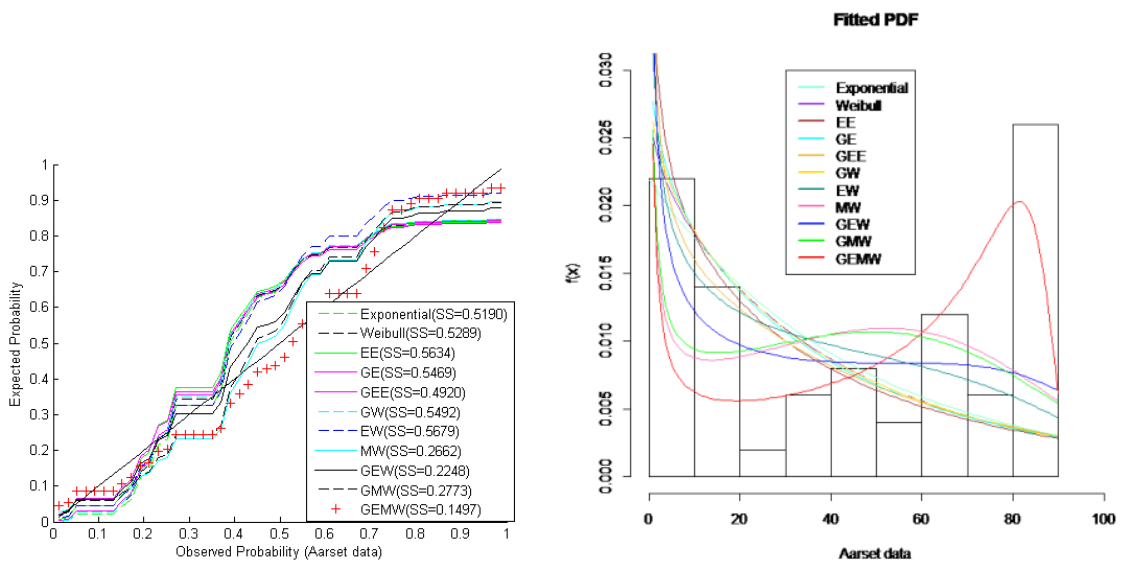

Figure 5: Fitted pdf and observed probabilities for Aarset data

The estimated covariance matrix for the GEMW distribution (Meeker and Escobar Data) is given by

$$
\left[\begin{array}{ccccc}
4.3338 E-9 & 7.134-6 & -1.9 E-7 & -1.37 E-6 & 5.384 E-8 \\
7.134 E-6 & 0.01184 & -0.00012 & -0.00029 & 0.000069 \\
-1.9 E-7 & -0.00012 & 0.000535 & 0.003490 & -0.00004 \\
-1.37 E-6 & -0.00029 & 0.000069 & 0.03409 & -0.00036 \\
5.384 E-8 & 0.000069 & -0.00004 & -0.00036 & 5.657 E-6
\end{array}\right]
$$

The $95 \%$ asymptotic confidence intervals for the GEMW model (Meeker and Escobar Data) parameters are: $\alpha \in(0.000328,0.09495), \beta \in(0.02917,0.03890), \delta \in(0.06499,0.8203), k \in$ (5.3111, 5.7562), and $\lambda \in(1567.009871,1567.010129)$, respectively.

Plots of the fitted densities, the histogram of these data and probability plots are presented in Figure 5 and Figure 6 . For the probability plot, we plotted $\mathrm{G}_{G E M W}\left(x_{(J)} ;(\hat{\alpha}, \hat{\beta}, \hat{k} . \hat{\lambda} . \hat{\delta})\right)$ against $\frac{j-0.375}{n+0.25}, j=1,2, \ldots, n$, where $x_{(J)}$ are the ordered values of the observed data.

For the Aarset data, the LR test statistic of the hypotheses $H_{0}$ : GEW against $H_{a}$ : GEMW and $H_{0}$ : GMW against $H_{a}$ : GEMW are $\omega_{1}=17.5$ with p-value $=2.87 \times 10^{-5}$ and $\omega_{2}=20.2$ with p-value $=6.98 \times 10^{-6}$. Therefore, we reject $H_{0}$ in favor of $H_{a}$ and conclude that the GEMW distribution is a significant better than the GEW and GMW distributions. Moreover, the values of the statistics AIC, AICC, BIC and KS show that model GEMW is a "better" fit for this data. Also, the value of SS for the GEMW distribution is the smallest.

Similarly, we can also conduct the LR test for the Meeker and Escobar data 

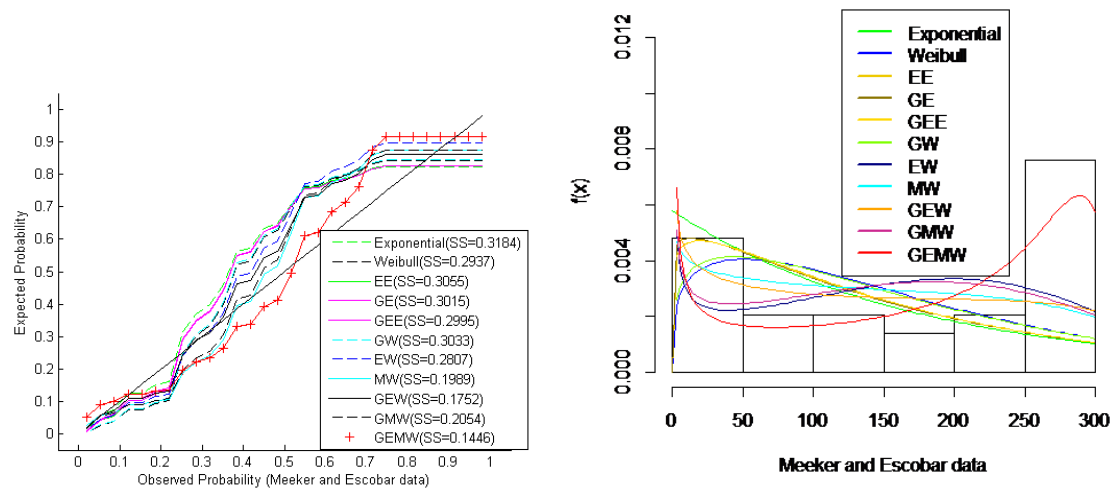

Figure 6: Fitted pdf and observed probabilities for Meeker and Escobar data

for the hypotheses $H_{0}$ : GEW against $H_{\mathrm{a}}$ : GEMW and $H_{0}$ : GMW against $H_{\mathrm{a}}$ : GEMW. The LR test statistic of these hypothesis are $\omega_{3}=10.8$ with $p$-value $=0.001$ and $\omega_{4}=14$ with $p$-value $=$ $1.83 \times 10^{-4}$, which implies that we should reject $H_{0}$ in favor of $H_{a}$ and conclude that the GEMW distribution is a significant better fit for the Meeker and Escobar data. In addition, the values of the statistics AIC, AICC, BIC and KS clearly show that model GEMW is a "better" fit for this data. Furthermore, the value of SS for the GEMW distribution is the smallest.

\section{Concluding Remarks}

A new class of distributions called the gamma-exponentiated or generalized modified Weibull (GEMW) distribution is proposed and studied. The GEMW distribution has several sub-models such as the GEW, EW, EE, GW, GE, GEE, GER, GR, MW, GEV, EV, GMW, Weibull, Rayleigh and exponential distributions as special cases. The density of this new class of distributions can be expressed as a linear combination of MW density functions. The GEMW distribution possesses hazard function with flexible behavior. We also obtain closed form expressions for the moments, distribution of order statistics and entropy. Maximum likelihood estimation technique is used to estimate the model parameters. Finally, the GEMW model is fitted to real data sets to illustrate the usefulness, flexibility and applicability of this class of distributions. 


\section{References}

[1] Aarset, M.V. (1987). How to identify bathtub hazard rate, IEEE: Transactions on Reliability, 36(1), 106-108.

[2] Bourguignon, M., Silva, R. B., Cordeiro, G. M. (2014). The Weibull-G family of probability distributions, Journal of Data Science, 12, 53-68.

[3] Carrasco, M., Ortega, E.M., Cordeiro, G.M. (2008). A generalized modified Weibull distribution for lifetime modeling, Computational Statistics and Data Analysis, 53(2), 450-462.

[4] Coles, S. (2001). An introduction to statistical modeling of extreme values, Springer Series in Statistics, London, Springer-Verlag.

[5] Eugene, N., Lee, C., Famoye, F. (2002). Beta-normal distribution and its applications, Communication in Statistics. Theory and Methods, 31, 497-512.

[6] Fergusen, T.A. (1996). A course in large sample theory, Chapman and Hall.

[7] Gupta, R.D., and Kundu, D. (1999). Generalized exponential distributions, Australian and New Zealand Journal of Statistics, 41, 173-188.

[8] Gradshteyn, I.S., Ryzhik, I.M. (2000). Tables of integrals. Series and Products,Academic Press: San Diego.

[9] Gupta, R.D., Kundu, D. (2001). Exponentiated exponential distribution: an alternative to gamma and Weibull distributions, Biometrical Journal, 43, 117-130.

[10]Haupt, E., Schabe, H. (1992). A new model for a lifetime distribution with bathtub shaped failure rate, Microelectronics and Reliability, 32, 633-639.

[11]Hjorth, U. (1980). A reliability distribution with increasing, decreasing, constant and bathtub failure rates, Technometrics, 22, 99-107.

[12]Jones, M.C. (2004). Families of distributions arising from distributions of order statistics, TEST, 13, 1-43.

[13]Kundu, D., Rakab, M.Z. (2005). Generalized Rayleigh distribution: different methods of estimation, Computational Statistics and Data Analysis, 49, 187200.

[14]Lai, C.D., Xie, M., Murthy, D.N.P. (2003). A modified Weibull distribution, IEEE Transactions on Reliability, 52, 33-37.

[15] Meeker, W.Q., Escobar, L.A. (1998). Statistical methods for reliability data, New York: John Wiley. 
[16]Merovci, F., Elbatal, I. (2015). A new generalization of exponentiated modified Weibull Distribution, Journal of Data Science, 13(2), 213-240.

[17]Nadarajah, S., Cordeiro, G.M., Ortega, E.M.M. (2011). General results for the beta-modified Weibull distribution, Journal of Statistical Computation and Simulation, 81(10), 1211-1232.

[18]Nadarajah, S., Kotz, S. (2006). The beta exponential distribution, Reliability Engineering and System Safety, 91, 689-697.

[19]Nelson, W. (1982). Lifetime data analysis, Wiley, New York.

[20]Pham, H., Lai, C.D. (2007). On recent generalizations of the Weibull distribution, IEEE, Transaction on Reliability, 56, 454-458.

[21]Pinho, G.B., Cordeiro, G.M., Nobre, J.S. (2012). The gamma-exponentiated Weibull distribution, Journal of Statistical Theory and Applications, 11(4), 379395.

[22]R Development Core Team, (2011). A Language and Environment for Statistical Computing, $R$ Foundation for Statistical Computing, Vienna, Austria.

[23]Rajarshi, S., Rajarshi, M.B. (1988). Bathtub distributions: A review, Communications in Statistics-Theory and Methods, 17, 2521-2597.

[24]Ristić, M.M., Balakrishnan, N. (2011). The gamma-exponentiated exponential distribution, Journal of Statistical Computation and Simulation, 82(8), 1191 1206.

[25]Shaked, M., Shanthikumar, J.G. (1994). Stochastic orders and their applica- tions, New York, Academic Press.

[26]Silva, G.O., Ortega, E.M.M., Cordeiro, G.M. (2010). The beta modified Weibull distribution, Lifetime Data Analysis, 16, 409-430.

[27]Seregin, A. (2010). Uniqueness of the maximum likelihood estimator for kmonotone densities, Proceedings of the American Mathematical Society, 10(138), 4511-4515.

[28]Santos-Silva. J.M.C., Tenreyro, S. (2010). On the existence of maximum likelihood estimates in Poisson regression, Economics Letters, 107, 310-312.

[29]Zhou, C. (2009). Existence and Consistency of the maximum likelihood estimator for the extreme index, Journal of Multivariate Analysis, 100, 794-815.

[30]Xia, J., Mi, J., Zhou, Y.Y. (2009). On the existence and uniqueness of the maximum likelihood estimators of normal and log-normal population parameters with grouped data, Journal of Probability and Statistics, Article id 310575, 16 pages. 
[31]Zografos, K., Balakrishnan, N. (2009). On families of beta and generalized gamma-generated distribution and associated inference, Statistical Methodology, 6, 344-362.

[32]Weibull, W. (1951). Statistical distribution function of wide applicability, Journal of Applied Mechanics, 18, 293-296. 


\section{APPENDIX}

Elements of the observed information matrix can be readily obtained from the second and mixed partial derivative given below:

$$
\begin{aligned}
& \frac{\partial^{2} \ell}{\partial \beta^{2}}=-\frac{x^{2}}{(k+\beta x)^{2}}+\frac{x^{2}\left(\frac{x}{\lambda}\right)^{k} e^{\beta x}\left(\alpha-e^{\left(\frac{x}{\lambda}\right)^{k} e^{\beta x}}\right)}{e^{\left(\frac{x}{\lambda}\right)^{k} e^{\beta x}}-1} \\
& -\frac{x^{2}(\alpha-1)\left(\frac{x}{\lambda}\right)^{2 k} e^{2 \beta x+\left(\frac{x}{\lambda}\right)^{k} e^{\beta x}}}{\left[1-e^{\left(\frac{x}{\lambda}\right)^{k} e^{\beta x}}\right]^{2}}-\frac{x^{2}(\delta-1)\left(\frac{x}{\lambda}\right)^{2 k} e^{2 \beta x}}{\left(1-e^{\left(\frac{x}{x}\right)^{k} e^{\beta x}}\right)^{2}\left(\log \left[1-e^{-\left(\frac{x}{\lambda}\right)^{k} e^{\beta x}}\right]\right)^{2}} \\
& \left.-\frac{x^{2}(\delta-1)\left(\frac{x}{\lambda}\right)^{k} e^{\beta x}\left(1-e^{\left(\frac{x}{\lambda}\right)^{k} e^{\beta x}}+\left(\frac{x}{\lambda}\right)^{k} e^{\beta x+\left(\frac{z}{\lambda}\right)^{k} e^{\beta x}}\right)}{\left(1-e^{\left(\frac{\pi}{x}\right)^{k} e^{\beta z}}\right)^{2}\left(\log \left[1-e^{-\left(\frac{\pi}{\lambda}\right)^{k} e^{\beta z}}\right]\right)^{2}}\right) . \\
& \frac{\partial^{2} \ell}{\partial \beta \partial \alpha}=\frac{x\left(\frac{x}{\lambda}\right)^{k} e^{\beta x}}{e^{\left(\frac{x}{\lambda}\right)^{k} e^{\beta x}}-1} \\
& \frac{\partial^{2} \ell}{\partial \beta \partial k}=-\frac{x}{(k+\beta x)^{2}}-x\left(\frac{x}{\lambda}\right)^{k} e^{\beta x} \log \left(\frac{x}{\lambda}\right)+\frac{x(\alpha-1)\left(\frac{x}{\lambda}\right)^{k} \log \left(\frac{x}{\lambda}\right) e^{\beta x}}{e^{\left(\frac{x}{\lambda}\right)^{k} e^{\beta x}}-1} \\
& -\frac{x(\alpha-1)\left(\frac{x}{\lambda}\right)^{2 k} \log \left(\frac{x}{\lambda}\right) e^{2 \beta x+\left(\frac{x}{x}\right)^{k} e^{\beta x}}}{\left(1-e^{\left(\frac{z}{x}\right)^{k} e^{\beta z}}\right)^{2}}+\frac{x(\delta-1)\left(\frac{x}{\lambda}\right)^{k} \log \left(\frac{x}{\lambda}\right) e^{\beta x}}{\left(e^{\left(\frac{z}{\lambda}\right)^{k} e^{\beta x}}-1\right) \log \left[1-e^{-\left(\frac{x}{\lambda}\right)^{k} e^{\beta x}}\right]} \\
& -\frac{x(\delta-1)\left(\frac{x}{\lambda}\right)^{2 k} \log \left(\frac{x}{\lambda}\right) e^{2 \beta x-\left(\frac{z}{\lambda}\right)^{k} e^{\beta z}}}{\left(1-e^{-(z)^{k} e^{\beta z}}\right)\left(e^{\left(\frac{x}{\lambda}\right)^{k} e^{\beta z}}-1\right)\left(\log \left[1-e^{-\left(\frac{z}{\lambda}\right)^{k} e^{\beta x}}\right]\right)^{2}} \\
& -\frac{x(\delta-1)\left(\frac{x}{\lambda}\right)^{2 k} \log \left(\frac{x}{\lambda}\right) e^{2 \beta x+\left(\frac{z}{\lambda}\right)^{k} e^{\beta z}}}{\left(1-e^{\left(\frac{x}{\lambda}\right)^{k} e^{\beta x}}\right)^{2} \log \left[1-e^{-\left(\frac{z}{\lambda}\right)^{k} e^{\beta x}}\right]} . \\
& \frac{\partial^{2} \ell}{\partial \beta \partial \lambda}=\frac{k x^{k+1} e^{\beta x}}{\lambda^{k+1}}-\frac{k x^{k+1}(\alpha-1) e^{\beta x}}{\lambda^{k+1}\left(e^{\left(\frac{x}{\lambda}\right)^{k} e^{\beta x}}-1\right)}+\frac{k x^{2 k+1}(\alpha-1) e^{2 \beta x+\left(\frac{z}{\lambda}\right)^{k} e^{\beta x}}}{\lambda^{2 k+1}\left(e^{\left(\frac{x}{\lambda}\right)^{k} e^{\beta x}}-1\right)^{2}} \\
& -\frac{k x^{k+1}(\delta-1) e^{\beta x}}{\lambda^{k+1}\left(e^{\left(\frac{x}{\lambda}\right)^{k} e^{\beta x}}-1\right) \log \left[1-e^{-\left(\frac{z}{\lambda}\right)^{k} e^{\beta x}}\right]} \\
& +\frac{k x^{2 k+1}(\delta-1) e^{2 \beta x-\left(\frac{z}{\lambda}\right)^{k} e^{\beta x}}}{\lambda^{2 k+1}\left(1-e^{-\left(\frac{z}{\lambda}\right)^{k} e^{\beta x}}\right)\left(e^{\left(\frac{x}{\lambda}\right)^{k} e^{\beta x}}-1\right)\left(\log \left[1-e^{-\left(\frac{x}{\lambda}\right)^{k} e^{\beta x}}\right]\right)^{2}} \\
& +\frac{k x^{2 k+1}(\delta-1) e^{2 \beta x+\left(\frac{x}{\lambda}\right)^{k} e^{\beta x}}}{\lambda^{2 k+1}\left(1-e^{\left(\frac{x}{\lambda}\right)^{k} e^{\beta x}}\right)^{2} \log \left[1-e^{-\left(\frac{z}{\lambda}\right)^{k} e^{\beta x}}\right]} . \\
& \frac{\partial^{2} \ell}{\partial \beta \partial \delta}=\frac{x\left(\frac{x}{\lambda}\right)^{k} e^{-\left(\frac{x}{\lambda}\right)^{k} e^{\beta x}+\beta x}}{\left(\log \left(1-e^{-\left(\frac{Z}{\lambda}\right)^{k} e^{\beta x}}\right)\right)\left(1-e^{-(\mathbb{X})^{k} e^{\beta x}}\right)} . \\
& \frac{\partial^{2} \ell}{\partial \alpha^{2}}=-\frac{\delta}{\alpha^{2}} . \quad \frac{\partial^{2} \ell}{\partial \alpha \partial k}=\frac{\left(\frac{x}{\lambda}\right)^{k} \log \left(\frac{x}{\lambda}\right) e^{\beta x}}{e^{\left(\frac{x}{\lambda}\right)^{k} e^{\beta z}}-1} . \\
& \frac{\partial^{2} \ell}{\partial \alpha \partial \lambda}=\frac{k x^{k} \lambda^{-k-1} e^{\beta x}}{1-e^{(\mathbb{X})^{k} e^{\beta z}}} . \quad \frac{\partial^{2} \ell}{\partial \alpha \partial \delta}=\frac{1}{\alpha} .
\end{aligned}
$$




$$
\begin{aligned}
\frac{\partial^{2} \ell}{\partial k^{2}}= & -\frac{1}{(k+\beta x)^{2}}-k k_{1}+k k_{2} \\
& +\frac{\left(\frac{x}{\lambda}\right)^{k} e^{\beta x}\left(\delta-1+\left(\alpha-e^{\left(\frac{x}{X}\right)^{k} e^{\beta x}}\right) \log \left[1-e^{-\left(\frac{x}{\lambda}\right)^{k} e^{\beta x}}\right]\right)\left[\log \left(\frac{x}{\lambda}\right)\right]^{2}}{\left(e^{\left(\frac{x}{\lambda}\right)^{k} e^{\beta x}}-1\right) \log \left[1-e^{-\left(\frac{x}{\lambda}\right)^{k} e^{\beta x}}\right]} \\
& -\frac{\left(\frac{x}{\lambda}\right)^{2 k} e^{2 \beta x+(\mathbb{x})^{k} e^{\beta x}}\left(\delta-1+\left(\alpha-e^{\left(\frac{x}{\lambda}\right)^{k} e^{\beta x}}\right) \log \left[1-e^{-(\mathbb{x})^{k} e^{\beta z}}\right]\right)\left[\log \left(\frac{x}{\lambda}\right)\right]^{2}}{\left(e^{\left(\frac{x}{\lambda}\right)^{k} e^{\beta x}}-1\right)^{2} \log \left[1-e^{-\left(\frac{z}{\lambda}\right)^{k} e^{\beta x}}\right]},
\end{aligned}
$$

where

$$
\begin{gathered}
k k_{1}=\frac{\left(\frac{x}{\lambda}\right)^{2 k} e^{2 \beta x-\left(\frac{x}{\lambda}\right)^{k} e^{\beta x}}\left(\delta-1+\left(\alpha-e^{\left(\frac{x}{\lambda}\right)^{k} e^{\beta x}}\right) \log \left[1-e^{-\left(\frac{x}{\lambda}\right)^{k} e^{\beta z}}\right]\right)\left[\log \left(\frac{x}{\lambda}\right)\right]^{2}}{\left(1-e^{-\left(\frac{x}{\lambda}\right)^{k} e^{\beta z}}\right)\left(e^{\left(\frac{x}{\lambda}\right)^{k} e^{\beta x}}-1\right)\left(\log \left[1-e^{-\left(\frac{z}{\lambda}\right)^{k} e^{\beta x}}\right]\right)^{2}}, \\
k k_{2}=\frac{k k_{2_{1}}}{\left(e^{\left(\frac{x}{\lambda}\right)^{k} e^{\beta x}}-1\right) \log \left[1-e^{\left.-\left(\frac{x}{\lambda}\right)^{k} e^{\beta x}\right]}\right.}, \\
k k_{2_{1}}=\frac{\left(\frac{x}{\lambda}\right)^{k} \log \left(\frac{x}{\lambda}\right) e^{\beta x-\left(\frac{z}{\lambda}\right)^{k} e^{\beta x}}\left(\alpha-e^{\left(\frac{z}{\lambda}\right)^{k} e^{\beta x}}\right)}{1-e^{-\left(\frac{x}{\lambda}\right)^{k} e^{\beta x}}\left(\frac{x}{\lambda}\right)^{k} \log \left(\frac{x}{\lambda}\right)} \\
-\left(\frac{x}{\lambda}\right)^{k} e^{\beta x+\left(\frac{x}{\lambda}\right)^{k} e^{\beta x}} \log \left(\frac{x}{\lambda}\right) \log \left[1-e^{-\left(\frac{x}{\lambda}\right)^{k} e^{\beta x}}\right] e^{\beta x}\left(\frac{x}{\lambda}\right)^{k} \log \left(\frac{x}{\lambda}\right) . \\
\frac{\partial^{2} \ell}{\partial \lambda^{2}}=\frac{\lambda_{1}}{\lambda_{2}},
\end{gathered}
$$

where

$$
\begin{gathered}
\lambda_{1}=k\left((1-\delta) k\left(\frac{x}{\lambda}\right)^{2 k} e^{2 \beta x}+(1-\delta) e^{\beta x}\left(1+k-(k+1) e^{\left(\frac{x}{\lambda}\right)^{k} e^{\beta x}}\right.\right. \\
\left.\left.+k\left(\frac{x}{\lambda}\right)^{k} e^{\beta x+\left(\frac{x}{\lambda}\right)^{k} e^{\beta x}}\right)\left(\frac{x}{\lambda}\right)^{k} \log \left[1-e^{-\left(\frac{z}{\lambda}\right)^{k} e^{\beta x}}\right]+\lambda_{11} \log \left[1-e^{-\left(\frac{x}{\lambda}\right)^{k} e^{\beta z}}\right]\right), \\
\lambda_{1_{1}}=1-2 e^{\left(\frac{x}{\lambda}\right)^{k} e^{\beta z}}+e^{2\left(\frac{z}{\lambda}\right)^{k} e^{\beta z}}-e^{\beta x+2\left(\frac{x}{\lambda}\right)^{k} e^{\beta x}}(1+k)\left(\frac{x}{\lambda}\right)^{k} \\
-(k+1) \alpha\left(\frac{x}{\lambda}\right)^{k} e^{\beta x}+(\alpha+1)(k+1)\left(\frac{x}{\lambda}\right)^{k} e^{\beta x+\left(\frac{x}{\lambda}\right)^{k} e^{\beta x}} \\
+k(1-\alpha)\left(\frac{x}{\lambda}\right)^{k} e^{2 \beta x+2\left(\frac{x}{\lambda}\right)^{k} e^{\beta z}}, \\
\lambda_{2}=\lambda\left(e^{\left(\frac{z}{\lambda}\right)^{k} e^{\beta x}}-1\right) \log \left[1-e^{-\left(\frac{x}{\lambda}\right)^{k} e^{\beta x}}\right] . \\
\frac{\partial^{2} \ell}{\partial k \partial \lambda}=\frac{\lambda_{k 1}}{\lambda_{k 2}},
\end{gathered}
$$

where 


$$
\begin{aligned}
\lambda_{k 1}= & (\delta-1) k\left(\frac{x}{\lambda}\right)^{2 k} e^{2 \beta x} \log \left(\frac{x}{\lambda}\right)+(\delta-1) e^{\beta x}\left(\frac{x}{\lambda}\right)^{k} \log \left[1-e^{-\left(\frac{x}{\lambda}\right)^{k} e^{\beta x}}\right] \\
& \times\left(1-e^{\left(\frac{x}{\lambda}\right)^{k} e^{\beta x}}+k\left(1-e^{\left(\frac{x}{\lambda}\right)^{k} e^{\beta x}}+\left(\frac{x}{\lambda}\right)^{k} e^{\beta x+\left(\frac{x}{\lambda}\right)^{k} e^{\beta x}}\right) \log \left(\frac{x}{\lambda}\right)\right) \\
+ & \left(\log \left[1-e^{-\left(\frac{x}{\lambda}\right)^{k} e^{\beta x}}\right]\right)^{2} \lambda_{k 11}, \\
\lambda_{k 1_{1}=}= & \left(e^{\left(\frac{x}{\lambda}\right)^{k} e^{\beta x}}-1\right)\left(1-e^{\left(\frac{x}{\lambda}\right)^{k} e^{\beta x}}+\left(\frac{x}{\lambda}\right)^{k} e^{\beta x+\left(\frac{x}{\lambda}\right)^{k} e^{\beta x}}-\alpha\left(\frac{x}{\lambda}\right)^{k} e^{\beta x}\right) \\
& +k\left(\frac{x}{\lambda}\right)^{k} \log \left(\frac{x}{\lambda}\right) e^{\beta x}\left[\alpha+e^{2\left(\frac{x}{\lambda}\right)^{k} e^{\beta x}}-(\alpha+1) e^{\left(\frac{x}{\lambda}\right)^{k} e^{\beta x}}\right] \\
& +k\left(\frac{x}{\lambda}\right)^{k} \log \left(\frac{x}{\lambda}\right) e^{\beta x}(\alpha-1)\left(\frac{x}{\lambda}\right)^{k} e^{\beta x+\left(\frac{x}{\lambda}\right)^{k} e^{\beta x}}
\end{aligned}
$$

and

$$
\begin{gathered}
\lambda_{k 2}=\lambda\left(e^{\left(\frac{x}{\lambda}\right)^{k} e^{\beta x}}-1\right)^{2}\left(\log \left[1-e^{-\left(\frac{x}{\lambda}\right)^{k} e^{\beta x}}\right]\right)^{2} . \\
\frac{\partial^{2} \ell}{\partial k \partial \delta}=\frac{\left(\frac{x}{\lambda}\right)^{k} \log \left(\frac{x}{\lambda}\right)}{\left(\log \left(1-e^{-\left(\frac{x}{\lambda}\right)^{k} e^{\beta z}}\right)\right)\left(1-e^{-\left(\frac{z}{\lambda}\right)^{k} e^{\beta x}}\right)} . \\
\frac{\partial^{2} \ell}{\partial \lambda \partial \delta}=\frac{-k x^{k} \lambda^{-k-1} e^{-\left(\frac{z}{\lambda}\right)^{k} e^{\beta x}+\beta x}}{\left(\log \left(1-e^{-\left(\frac{x}{\lambda}\right)^{k} e^{\beta x}}\right)\right)\left(1-e^{-\left(\frac{z}{\lambda}\right)^{k} e^{\beta z}}\right)} . \\
\frac{\partial^{2} \ell}{\partial \delta^{2}}=\frac{\left(\Gamma^{\prime}(\delta)\right)^{2}-\Gamma^{\prime \prime}(\delta) \Gamma(\delta)}{\Gamma^{2}(\delta)} .
\end{gathered}
$$


Shusen $\mathrm{Pu}$

Department of Mathematics, Applied Mathematics and Statistics

Case Western Reserve University, OH 44106, USA

shusen.pu@ case.edu

Broderick O. Oluyede

Department of Mathematical Sciences

Georgia Southern University, GA 30460, USA

boluyede@georgiasouthern.edu

Yuqi Qiu

Department of Family Medicine and Public Health

University of California, San Diego, CA 92093, USA

Daniel Linder

Department of Biostatistics

Georgia Southern University, GA 30460, USA 
614 A GENERALIZED CLASS OF EXPONENTIATED MODI ED WEIBULL DISTRIBUTION WITH APPLICATIONS 